\title{
Contribuição à análise do gerador de indução trifásico com rotor bobinado: estudos experimentais e teóricos em condições normais de operação e diante de desequilíbrios
}

\section{de tensão}

\author{
Contribution to the analysis of the three-phase induction generator with wound rotor: experimental \\ and theoretical studies under normal operating conditions and with unbalance voltage
}

Contribución al análisis del generador de inducción trifásico con rotor bobinado: estudios

experimentales y teóricos en condiciones normales de funcionamiento y ante desequilibrios de tensión

Recebido: 18/11/2021 | Revisado: 25/11/2021 | Aceito: 26/11/2021 | Publicado: 07/12/2021

\author{
Vinícius Marcos Pinheiro \\ ORCID: https://orcid.org/0000-0002-5922-1188 \\ Universidade Federal de Uberlândia, Brasil \\ E-mail: viniciusmarcospinheiro@ hotmail.com \\ Cássio Alves de Oliveira \\ ORCID: https://orcid.org/0000-0003-3050-9361 \\ Universidade Federal de Uberlândia, Brasil \\ E-mail: kass-07@hotmail.com \\ Luciano Coutinho Gomes \\ ORCID: https://orcid.org/0000-0002-2827-6944 \\ Universidade Federal de Uberlândia, Brasil \\ E-mail: lcgomes@ufu.br
}

\begin{abstract}
Resumo
No presente trabalho é analisado o comportamento da máquina de indução trifásica de rotor bobinado, muito empregada na matriz eólica, operando como gerador em condições normais e com desequilíbrios de tensão no sistema elétrico em que está conectado. Para isso, é adotado um modelo matemático da máquina de indução trifásica denominado "modelo na fase", em que não são realizadas transformações de coordenadas ou de sistema de referência, além de levar em consideração os efeitos da saturação magnética. Os resultados provenientes desse modelo, que foi implementado computacionalmente, são comparados com os resultados obtidos em uma plataforma experimental de ensaios. Em ambos os casos foram obtidas as curvas de tensão e corrente que permitiram, por meio de comparação, verificar a resposta satisfatória do modelo matemático frente ao distúrbio analisado. A partir disso, foi possível concluir que o modelo é adequado para estudos em condições normais de operação e com desequilíbrios de tensão.

Palavras-chave: Gerador de indução; Plataforma experimental de ensaios; Matriz eólica; Rotor bobinado.
\end{abstract}

\begin{abstract}
In the present work analyzes the behavior of the three-phase induction machine with a wound rotor, which is widely used in the wind energy systems, operating as a generator under normal conditions and with unbalance voltage in the electrical system to which it is connected. For this, a mathematical model of the three-phase induction machine called "phase model" is adopted, in which coordinate or reference system transformations are not performed, in addition to taking into account the effects of magnetic saturation. The results from this model, which was implemented computationally, are compared with the results obtained in an experimental test platform. In both cases, the voltage and current curves were obtained, this data allowed, by means of comparison, to verify the satisfactory response of the mathematical model regarding the analyzed disturbance. From this, it was possible to conclude that the model is suitable for studies under normal operating conditions and with unbalance voltage.
\end{abstract}

Keywords: Induction generator; Experimental test platform; Wind energy systems; Wound rotor.

\section{Resumen}

En el presente trabajo analiza el comportamiento de la máquina de inducción trifásica con rotor bobinado, muy utilizada en la matriz eólica, funcionando como generador en condiciones normales y con desequilibrios de tensión en el sistema eléctrico al que está conectada. Para ello, se adopta un modelo matemático de la máquina de inducción trifásica denominado "modelo en fase", en el que no se realizan transformaciones de coordenadas o sistemas de referencia, además de tener en cuenta los efectos de la saturación magnética. Los resultados de este modelo, que se 
implementó computacionalmente, se comparan con los resultados obtenidos en una plataforma de pruebas experimental. En ambos casos se obtuvieron curvas de tensión y corriente que permitieron, mediante comparación, verificar la respuesta satisfactoria del modelo matemático frente a la perturbación analizada. De esto se pudo concluir que el modelo es apto para estudios en condiciones normales de operación y con desequilibrios de voltaje.

Palabras clave: Generador de inducción; Plataforma de pruebas experimentales; Matriz eólica; Rotor bobinado.

\section{Introdução}

A máquina de indução surgiu, de forma comercial, no final do século XIX. De acordo com o antigo engenheiro chefe da Westinghouse Electric Company, Benjamin Garver Lamme (1921), apesar de os créditos da invenção serem atribuídos principalmente a Nikola Tesla, diversos outros pesquisadores à época estavam engajados em linhas de pesquisas que circundavam esse equipamento. Segundo Lamme, nomes como Galileo Ferraris, Oliver Blackburn Shallenberger e Elihu Thomson tiveram importantes contribuições nessa área, o que, para o respectivo autor, levaria à invenção da máquina de indução mais cedo ou mais tarde.

Existem dois tipos de máquina de indução, que se diferenciam pela forma que o rotor, parte móvel da máquina, é construído. Primeiramente, tem-se aquela conhecida como máquina de indução com rotor gaiola de esquilo (MIRG), a qual é amplamente utilizada como motor por ser robusta, de baixo custo, de fácil manutenção e elevada eficiência (Oliveira, 2018). Nesse caso, o condutor do rotor é formado por barras condutoras que são curto-circuitadas em suas extremidades (Fitzgerald et al., 2006), como pode ser visualizado na Figura 1, não sendo possível, assim, seu acesso de forma externa.

Figura 1 - Rotor gaiola de esquilo.

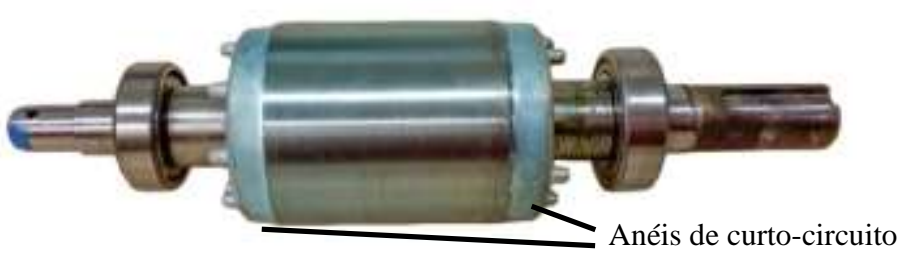

Fonte: Autores.

A outra, foco do presente trabalho, diz respeito à máquina de indução com rotor bobinado (MIRB), que possui sua aplicação voltada para a geração de eletricidade, principalmente, na matriz eólica. Tem-se, atualmente, duas topologias de geradores que empregam a MIRB, sendo elas: gerador de indução duplamente alimentado (Li et al., 2017; Zhou et al., 2018; Liu et al., 2019) que, segundo o Global Wind Energy Council-GWEC (GWEC, 2021), foi o gerador eólico mais vendido nos anos de 2017, 2018 e 2019; e o gerador com variação de resistência rotórica (El-Shimy, 2010). Conforme está apresentado na Figura 2, os terminais dos enrolamentos do rotor são conectados a anéis que se encontram sobre o eixo da máquina, permitindo seu acesso de forma externa. Esse acesso é realizado por meio de escovas de carvão que ficam em contato direto com os anéis, esclarecendo, desse modo, a maior necessidade de manutenção que essa topologia de máquina exige.

Assim sendo, o atual cenário é marcado por melhorias e desafios de forma a integrar, de maneira harmoniosa, a energia eólica à matriz energética, seja no Brasil, como nos demais países que estejam adotando essa promissora forma de geração de eletricidade. A necessidade por atender requisitos e procedimentos estabelecidos em documentos ou normas específicas (Lima, 2019) faz com que diversas pesquisas sejam desenvolvidas de forma a sanar ou mitigar problemas existentes, principalmente no que tange aos geradores empregados, buscando atender o consumidor da melhor maneira possível.

Diversos trabalhos envolvendo geradores de indução foram e continuam sendo desenvolvidos, podendo ser encontrados inúmeros documentos na literatura técnico-científica que analisam diversos aspectos relacionados a esse 
equipamento. Uma das metodologias mais recorrentes e eficientes empregada em tais trabalhos está relacionada às simulações computacionais dos modelos matemáticos que descrevem o funcionamento da máquina.

Figura 2 - Rotor bobinado.

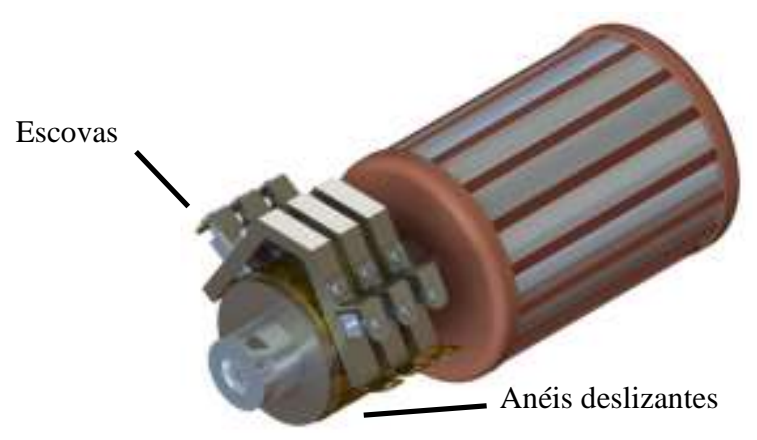

Fonte: Autores.

Neste contexto, em virtude da importância em se obter um modelo que represente o comportamento do gerador de indução trifásico de rotor bobinado da forma mais fidedigna possível, o presente trabalho tem como objetivo avaliar o modelo desenvolvido por Resende (1999) e Bispo et al. (2001) para tal fim, realizando comparações com estudos práticos, tendo sido desenvolvida e implementada, para isso, uma plataforma de ensaios, com o propósito de permitir a obtenção, de maneira precisa e confiável de dados tais como tensão, corrente e velocidade. Além disso, é analisado a resposta da máquina e do modelo matemático diante de desequilíbrios de tensão respeitando os limites estabelecidos no módulo 8 dos Procedimentos de Distribuição de Energia Elétrica no Sistema Nacional (PRODIST), elaborados pela Agência Nacional de Energia Elétrica (ANEEL).

\section{Metodologia}

A pesquisa aqui abordada é de caráter teórico-experimental, com abordagem quantitativa.

Para a análise do modelo matemático da máquina de indução trifásica operando como gerador, adotou-se o software MATLAB. No sentido de obter dados do funcionamento prático do gerador, uma plataforma de ensaios foi implementada para esse fim.

\subsection{Modelo matemático da máquina de indução trifásica no domínio da fase}

A modelagem da máquina empregando a mudança de referencial é um dos modelos mais utilizados e simples para se realizar estudos computacionais. Como exemplo, tem-se o trabalho desenvolvido por Vanço et al. (2018) na análise do gerador de indução de rotor do tipo gaiola de esquilo em geração distribuída, por meio de modelagem no referencial de eixo direto e em quadratura, levando em consideração o fenômeno da saturação do material magnético. Em (Santos et al., 2019) ocorre também o emprego do mesmo modelo para analisar o gerador de indução operando como gerador eólico conectado diretamente à rede.

Outros modelos mais complexos, em que não ocorrem a mudança de referencial, denominados por "modelo na fase", também podem ser encontrados. Em especial, tem-se o modelo desenvolvido por Resende (1999) e Bispo et. al. (2001) que, além de levar em consideração o fenômeno da saturação magnética, também inclui os harmônicos espaciais por considerar a fase da máquina como um enrolamento distribuído e de passo fracionário.

Resende (1999) emprega a modelagem na fase para realizar análises da máquina de indução trifásica como gerador auto excitado operando isoladamente. Por sua vez, Bispo et al. (2001) aplica o modelo para avaliar a atuação da máquina como 
motor. Outros trabalhos envolvendo o respectivo modelo da máquina podem ser encontrados na literatura técnico-científica. Freitas et al. (2003) emprega-o para auxiliar na análise dos efeitos da saturação magnética em motores de indução acionados por conversores. Ademais, Gomes (2008) realiza o estudo do acionamento vetorial do motor de indução com enfraquecimento de campo e maximização do conjugado por ampère, utilizando o respectivo modelo como auxílio.

O modelo no domínio da fase emprega o conceito de função harmônica magnética, em que são adotadas como variáveis de estado o concatenamento total de fluxo das fases, a velocidade e a posição angular. Diferentemente do modelo no domínio $d q$ (Abad et al., 2014), neste não ocorre a transformação de variáveis, o que torna seu equacionamento mais complexo, contudo, permite que fenômenos eletromagnéticos e variações dos parâmetros decorrentes da saturação do material magnético sejam representados de forma mais adequada, possibilitando uma melhor percepção física desses.

Este modelo considera as componentes fundamental e de terceiro harmônico da distribuição espacial de fluxo no entreferro no desenvolvimento matemático, por serem as componentes mais relevantes na distribuição espacial da força magnetomotriz, conforme está apresentado na Figura 3. As funções harmônicas magnéticas são obtidas de forma experimental por meio de medições das tensões e correntes terminais, conforme procedimento descrito mais adiante. Essas funções englobam os detalhes construtivos da máquina, tanto do circuito magnético quanto do enrolamento, além delas definirem os diversos pontos de operação da máquina.

Figura 3 - Distribuição espacial da força magnetomotriz.

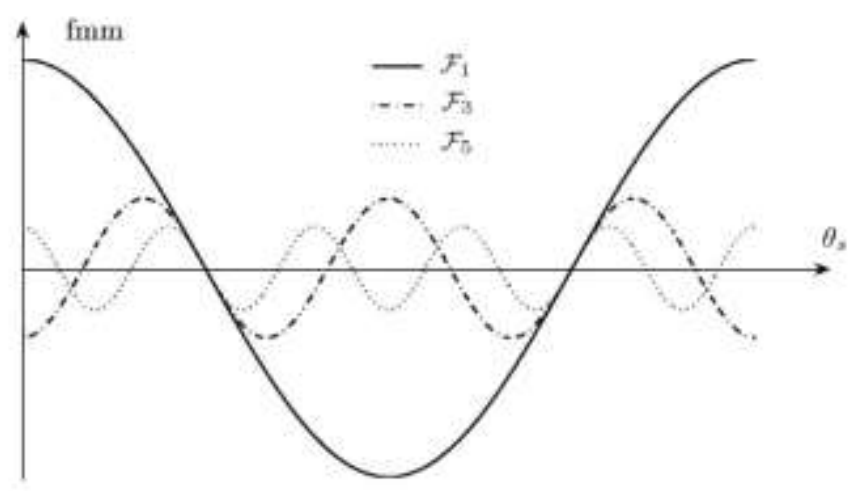

Fonte: Bim (2012).

Primeiramente, para uma fase genérica "n" da máquina de indução, seja de estator quanto de rotor, a tensão em seus terminais, que é dada pela soma da queda de tensão nas resistências dos seus enrolamentos devido ao efeito Joule, com a tensão decorrente da variação do fluxo magnético concatenado, pode ser escrita da seguinte forma

$$
v_{n}=R_{n} i_{n}+\frac{d \lambda_{n}}{d t}
$$

em que ${v_{n}}_{n}, i_{n}, R_{n}$ e $\lambda_{n}$, representam a tensão, corrente, resistência e concatenamento total de fluxo, respectivamente, para cada fase "n".

O concatenamento total de fluxo, considerando o fluxo de dispersão linear, é dado pela soma das componentes de dispersão, $\lambda_{d n}$, com a componente de magnetização, $\lambda_{m n}$, ou seja,

$$
\lambda_{n}=\lambda_{d n}+\lambda_{m n}=L_{n n} i_{n n}+\lambda_{m n}
$$


onde $L_{n n}$ é a indutância de dispersão.

Isolando em (2) o termo da corrente $\dot{i}_{n}$, chega-se então à seguinte equação:

$$
i_{n}=\frac{\lambda_{n}-\lambda_{m n}}{L_{n}}
$$

A equação (3) é substituída na equação (1) e, a partir das funções magnéticas, que são obtidas considerando apenas a componente fundamental da distribuição da força magnetomotriz resultante, é calculado o concatenamento de fluxo magnetizante e, posteriormente, a corrente e o fluxo total concatenado por fase. Dessa forma, sendo $v_{n}$ e $R_{n}$ valores conhecidos, é possível solucionar a equação (1).

Para concluir o desenvolvimento matemático, um sistema de referência deve ser adotado, conforme indicado na Figura 4. Nesse sistema, considera-se que a distribuição espacial de força magnetomotriz, $f m m(\theta)$, esteja localizada em sua totalidade no entreferro da máquina, em que é definido um eixo de referência $\theta$, cuja origem é a fase "a" do estator. Assim sendo, em um dado instante, o valor máximo da distribuição espacial de força magnetomotriz, $F M$, estará em uma posição angular $\alpha$.

Figura 4 - Distribuição espacial da força magnetomotriz.
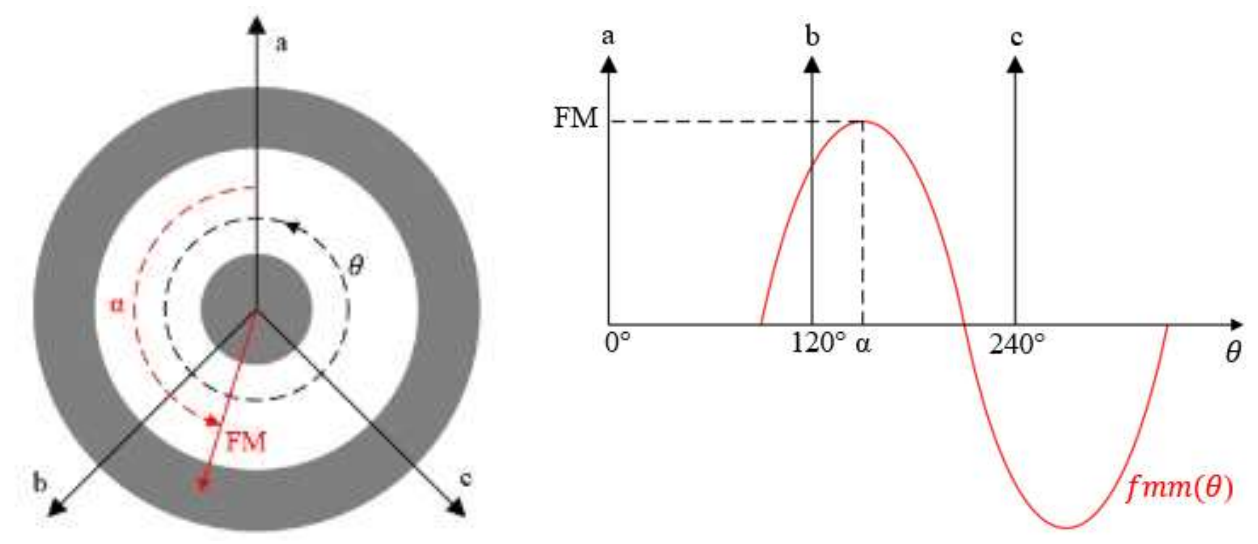

Fonte: Autores.

De acordo com o sistema de referência apresentado na Figura 4, a força magnetomotriz pode ser representada pela expressão

$$
f m m(\theta)=F M \cos (\theta-\alpha)
$$

Ainda segundo Resende (1999) e Bispo et al. (2001), a relação (4) pode ser igualada à expressão

$$
\sum_{n=a_{i}, b_{B}, A_{B}, C} 2 K_{n} i_{n} \cos \left(\theta-\theta_{n}\right)
$$

Em que:

$K_{n}-$ Fator de enrolamento das fases de estator e rotor. 
$\theta_{n}-$ Posição genérica das fases de estator e rotor.

$a, b, c-$ Fases a, b e c de estator.

$A, B, C-$ Fases $\mathrm{A}, \mathrm{B}$ e $\mathrm{C}$ de rotor.

A distribuição espacial da força magnetomotriz dará origem a uma distribuição de densidade de fluxo magnético, $B(\theta)$, conforme está apresentado na Figura 5. A densidade de fluxo terá uma forma não senoidal em fase com a curva $f m m(\theta)$.

Figura 5 - Distribuição espacial da força magnetomotriz e da densidade de fluxo magnético.

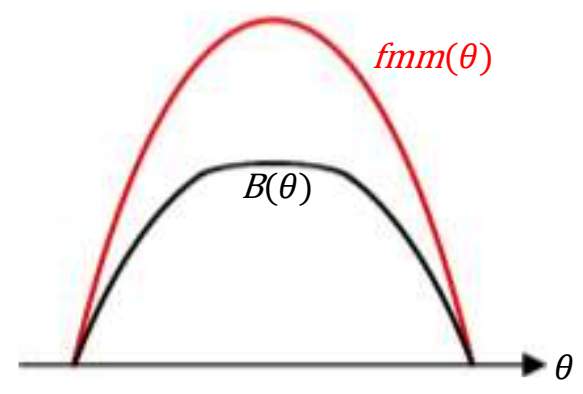

Fonte: Autores.

A decomposição da distribuição espacial de densidade de fluxo na série de Fourier permite escrever a seguinte equação:

$$
B(\theta)=\sum_{h=1, \mathbb{3}} B_{h} \cos [h(\theta-\alpha)]
$$

Onde:

$B_{h}-$ Valor máximo da densidade de fluxo de ordem $\mathrm{h}$.

$h$ - Ordem harmônica.

Logo, o fluxo magnetizante, $\lambda_{\operatorname{mm}}$, para uma fase genérica "n" devido à densidade de fluxo magnético $B(\theta)$ será dado por

$$
\lambda_{m n}=\sum_{h=1: a} F_{h}(\overline{F M}) \cos \left[h\left(\alpha-\theta_{n}\right)\right]
$$

em que $F_{h}(\overline{F M})$ é a função harmônica magnética e $\theta_{n}$ a posição genérica da fase "n".

As equações (1)-(7), quando manipuladas, permitem obter as seguintes expressões, as quais podem ser verificadas nas referências supracitadas.

$$
f_{R}(\lambda)=\sum_{n=a_{i}, b_{B}, A, B, C} \frac{\lambda_{n}}{L_{n}} \cos \left(\theta_{n}\right)
$$




$$
\begin{aligned}
& f_{i}(\lambda)=\sum_{n=\alpha_{B}, b_{B}, A_{B}, C} \frac{\lambda_{n}}{L_{n}} \operatorname{sen}\left(\theta_{n}\right) \\
& f(\lambda)^{2}=f_{R}(\lambda)^{2}+f_{I}(\lambda)^{2} \\
& A_{s}=\frac{3}{2}\left(\frac{1}{L_{g}}+\frac{1}{L_{g}}\right) \\
& F_{1}(\overline{F M})=\frac{f(\lambda)}{A_{g}}-\frac{\overline{F M}}{A_{g}} \\
& \operatorname{tg}(\alpha)=\frac{f_{I}(\lambda)}{f_{R}(\lambda)}
\end{aligned}
$$

Onde:

$\mathrm{L}_{\mathrm{g}}$ - Indutância de dispersão do estator.

$\mathrm{L}_{\mathrm{T}}$ - Indutância de dispersão do rotor.

Acionando a máquina de indução na velocidade síncrona, diversos valores eficazes de tensão balanceados e sem distorção são aplicados nos enrolamentos de estator e os valores instantâneos da tensão e corrente devem ser registrados. Subtraindo da corrente mensurada a componente relacionada com as perdas no ferro, obtém-se a corrente de magnetização, a qual permite determinar o concatenamento do fluxo de magnetização. Desse último, a partir da decomposição na série de Fourier, são obtidas as curvas das funções harmônicas magnéticas $F_{1}(\overrightarrow{F M})$ e $F_{2}(F M)$.

De posse das curvas das funções magnéticas, empregando-se um método de integração, pode-se, então, solucionar (1), em que, para cada passo de integração, os valores da tensão, $v_{n}$, e do concatenamento de fluxo total, $\lambda_{n}$, são conhecidos. Com os valores de $\lambda_{n}$ são calculadas as variáveis $f_{R}(\lambda)$ e $f_{i}(\lambda)$ por meio das equações (8) e (9) respectivamente, as quais possibilitam obter $f(\lambda)$ através de (10). A constante $A_{g}$ é obtida por meio de (11). Determinadas todas essas grandezas, a equação de uma reta é então definida por (12), cuja intersecção com a curva $F_{1}(F M)$ versus $\overline{F M}$, conforme está representada pela Figura 6, fornece os valores de $F_{1}(\overrightarrow{F M})$ e $\overline{F M}$ para cada passo de integração. Posteriormente, os valores de $F_{a}(F M)$ são obtidos por meio de interpolação numérica com os valores de $F_{1}(\overrightarrow{F M})$.

Figura 6 - Esboço da interseção da função magnética de ordem fundamental com a curva de magnetização.

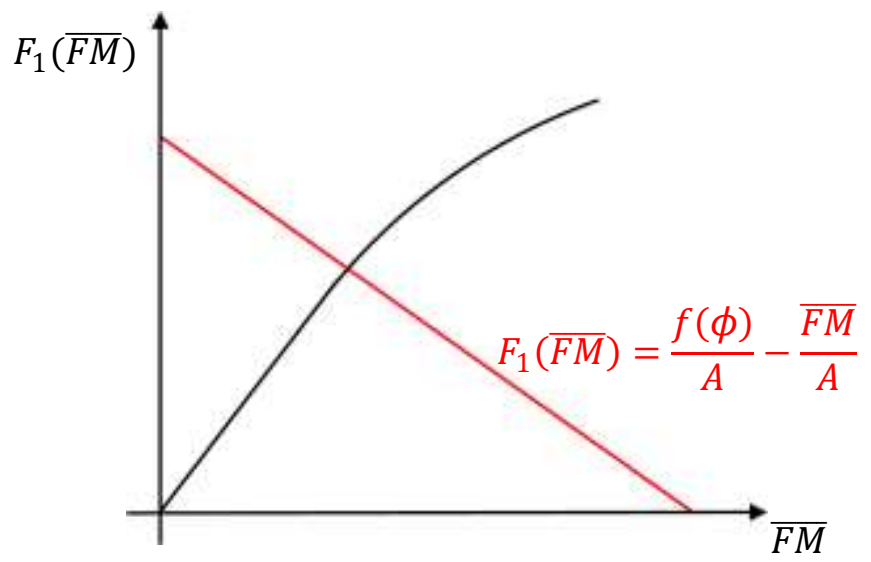

Fonte: Autores. 
Com os valores das funções harmônicas e de posse das correntes de rotor, o conjugado eletromagnético pode ser obtido por

$$
T_{e}=\frac{p}{2} \sum_{h=1 / a} F_{h}(F M) h[a+b+c]
$$

onde

$$
\begin{gathered}
a=i_{A} \operatorname{sen}\left(\alpha-\theta_{r}\right) \\
b=i_{B} \operatorname{sen}\left(\alpha-\theta_{r}-120^{\circ}\right) \\
c=i_{C} \operatorname{sen}\left(\alpha-\theta_{r}+120^{\circ}\right)
\end{gathered}
$$

em que:

$i_{A^{s}} i_{B^{s}} i_{C}-$ Correntes das fases A, B e C de rotor, respectivamente.

$\theta_{Y}-$ Posição angular do rotor.

A equação que rege a parte mecânica para a máquina operando como gerador é dada por:

$$
T_{B}-T_{m}-D \cdot \omega_{R}=J \cdot \frac{d \omega_{R}}{d t}
$$

Onde:

$T_{m}$ - Conjugado eletromagnético.

$T_{B}$ - Conjugado da turbina ou conjugado imposto.

D - Coeficiente de atrito viscoso.

$\omega_{R}-$ Velocidade angular do rotor.

l- Momento de inércia das partes girantes.

$$
\omega_{R}=\frac{d \theta_{R}}{d t}
$$

\subsection{Bancada de ensaios}

A Figura 7 apresenta o desenho confeccionado no software SolidWorks®, que detalha os equipamentos que compõem a plataforma implementada. Para facilitar a descrição de seu funcionamento, os principais elementos da plataforma podem ser classificados da seguinte forma:

- Sistema motriz: este primeiro sistema diz respeito às máquinas elétricas rotativas que integram a plataforma, sendo elas a máquina de indução trifásica de rotor bobinado e a máquina de indução trifásica de rotor em gaiola.

- Sistema de alimentação e controle das máquinas elétricas rotativas: para o acionamento da máquina de indução de rotor em gaiola é utilizado um conversor de frequência, enquanto que a máquina de rotor bobinado é alimentada por uma fonte programável. 
- Sistema de condicionamento e aquisição de dados: diversos equipamentos são empregados para a obtenção e acompanhamento dos dados da máquina de indução de rotor bobinado, dentre os quais podem ser citados: a fonte programável, osciloscópios, tacômetro, multímetros digitais e módulos de condicionamento e aquisição dos sinais de tensão e corrente.

Figura 7 - Desenho da plataforma experimental implementada.

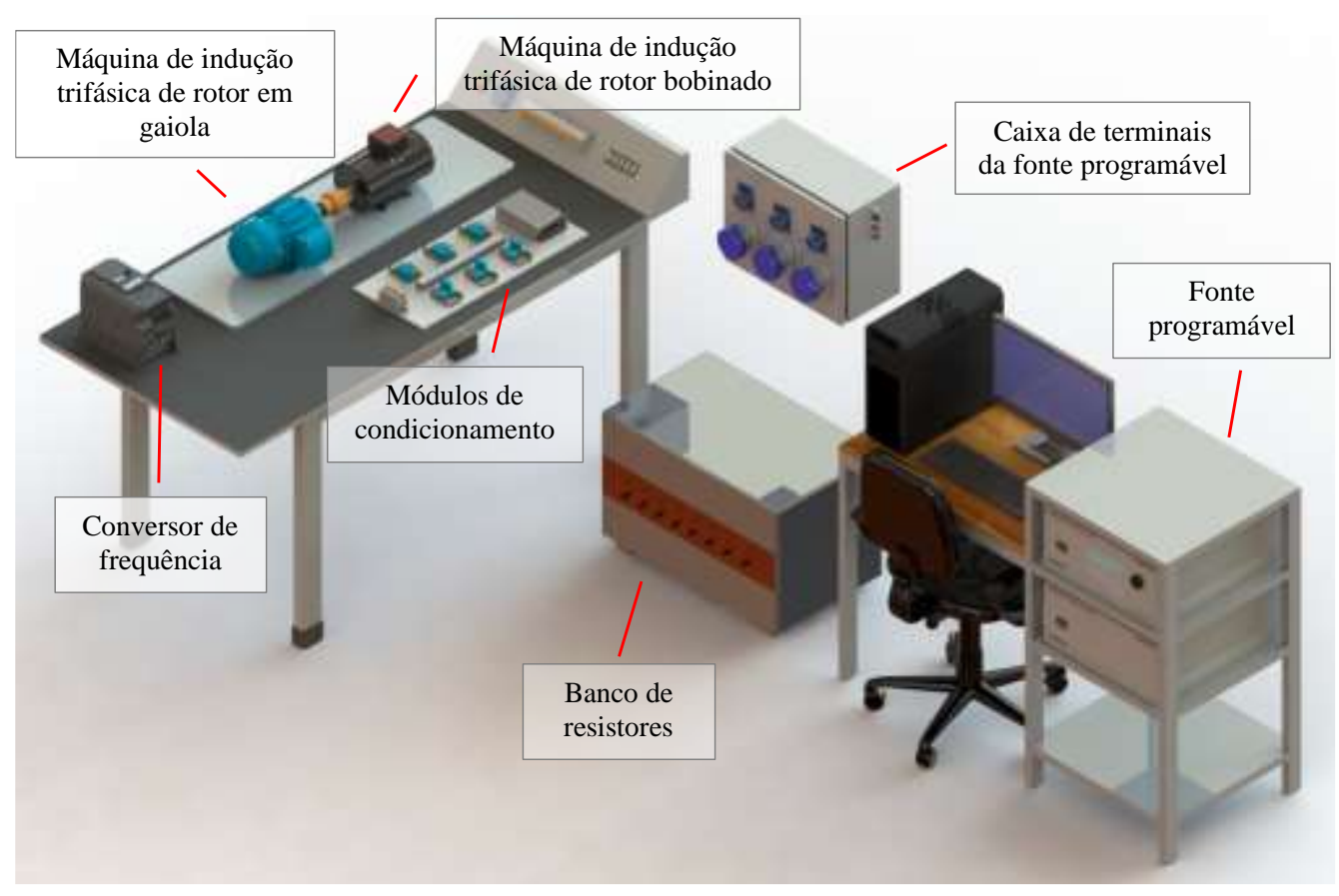

Fonte: Autores.

\subsubsection{Máquina de indução trifásica de rotor bobinado}

A máquina de indução trifásica de rotor bobinado analisada é um equipamento de $1 \mathrm{cv}$ que possui doze terminais acessíveis para o estator e três para o rotor (os enrolamentos de rotor são ligados internamente em estrela isolada). As principais características da máquina bem como os possíveis arranjos de ligação dos enrolamentos do estator estão indicados nas Tabelas 1 e 2 , respectivamente.

Tabela 1 - Principais características de placa da MIRB.

\begin{tabular}{cc}
\hline \multicolumn{2}{c}{ Máquina de indução trifásica - Rotor bobinado } \\
\hline Fabricante & MOTRON \\
\hline Frequência & $60 \mathrm{~Hz}$ \\
\hline Potência & $1 \mathrm{cv}$ \\
\hline Velocidade mecânica & $1750 \mathrm{rpm}$ \\
\hline Fator de potência & 0,6 \\
\hline Rendimento & $76 \%$ \\
\hline Classe de isolamento & $\mathrm{H}$ \\
\hline Regime de serviço & $\mathrm{S} 1$ \\
\hline
\end{tabular}

Fonte: Autores. 
Tabela 2 - Possíveis arranjos dos enrolamentos da MIRB.

\begin{tabular}{ccc}
\hline \multicolumn{3}{c}{ Possíveis arranjos dos enrolamentos do estator } \\
\hline Tipo & Tensão $(\mathbf{V})$ & Corrente $(\mathbf{A})$ \\
\hline Delta-Série & 440 & 1,95 \\
\hline Estrela-Série & 760 & - \\
\hline Delta-Paralelo & 220 & 3,9 \\
\hline Estrela-Paralelo & 380 & 2,3 \\
\hline
\end{tabular}

Fonte: Autores.

3.

Os parâmetros elétricos e mecânicos da máquina de indução trifásica de rotor bobinado estão apresentados na Tabela

Tabela 3 - Parâmetros elétricos e mecânicos da MIRB.

\begin{tabular}{ccc}
\hline Resistência de estator & $R_{g}$ & $8,116 \Omega$ \\
\hline Resistência de rotor & $R_{r}$ & $11,059 \Omega$ \\
\hline $\begin{array}{c}\text { Indutância de dispersão de } \\
\text { estator }\end{array}$ & $L_{g}$ & $18,456 \mathrm{mH}$ \\
\hline $\begin{array}{c}\text { Indutância de dispersão de } \\
\text { rotor }\end{array}$ & $L_{F}$ & $18,456 \mathrm{mH}$ \\
\hline Momento de inércia & $J$ & $13,2 \times 10^{-8} \mathrm{~kg} \cdot \mathrm{m}^{2}$ \\
\hline Coeficiente de atrito viscoso & $D$ & $0,491 \cdot 10^{-8} \mathrm{~kg} \cdot \mathrm{m}^{2} / \mathrm{s}$ \\
\hline
\end{tabular}

Fonte: Autores.

\subsubsection{Fonte programável}

A fim de alimentar a máquina de indução trifásica de rotor bobinado com tensões trifásicas senoidais e balanceadas, foi empregada uma fonte programável desenvolvida pela AMETEK Programmable Power®, modelo CSW5550. Esse equipamento, que está apresentado na Figura 8, possui dois módulos que fornecem, cada um, uma potência máxima de 1,85 kVA por fase. Além disso, outra característica que a torna fundamental para as análises realizadas é o baixo valor da distorção da tensão de saída, sendo de $0,25 \%$ para tensões que possuem uma frequência elétrica que esteja no intervalo de 40 a $100 \mathrm{~Hz}$.

Figura 8 - Fonte programável modelo CSW5550.

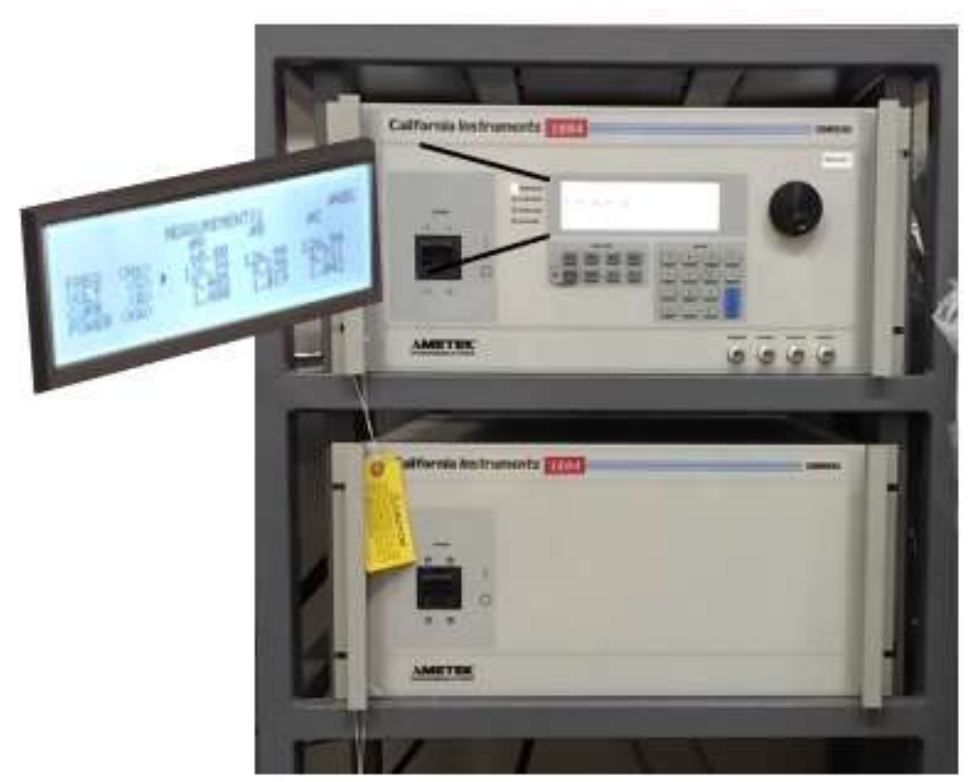

Fonte: Autores. 


\subsubsection{Emulador de turbina}

Com o intuito de analisar o funcionamento da máquina de indução de rotor bobinado como gerador, é acoplada em seu eixo uma máquina de indução trifásica de rotor gaiola de $3 \mathrm{cv}$, denominada aqui por máquina primária. As principais informações relativas a esse equipamento estão apresentadas na Tabela 4.

Tabela 4 - Dados de placa da máquina de indução trifásica de rotor do tipo gaiola de esquilo.

\begin{tabular}{cc}
\hline \multicolumn{2}{c}{ Máquina de indução trifásica - Rotor gaiola } \\
\hline Potência & $3 \mathrm{cV}$ \\
\hline Tensão $(\Delta, \mathbf{Y})$ & $220 / 380 \mathrm{~V}$ \\
\hline Corrente $(\Delta, \mathbf{Y})$ & $8,39 / 4,86 \mathrm{~A}$ \\
\hline Frequência & $60 \mathrm{~Hz}$ \\
\hline Velocidade mecânica & $3450 \mathrm{rpm}$ \\
\hline Categoria & $\mathrm{N}$ \\
\hline Fator de potência & 0,84 \\
\hline Fator de serviço & 1,15 \\
\hline Classe de isolamento & $\mathrm{F}$ \\
\hline
\end{tabular}

Fonte: Autores.

Para realizar o controle de velocidade da máquina de indução trifásica de rotor gaiola, é empregado um conversor de frequência modelo CFW09 da WEG. Nesse dispositivo estão disponíveis as seguintes formas de controle: escalar, vetorial sensorless e vetorial com encoder. Uma vez que a aplicação no qual ele está inserido não exige resposta dinâmica rápida, em razão da não variação brusca de carga, que no caso é o gerador, optou-se pela utilização do controle escalar. A velocidade no eixo é aferida através de um tacômetro digital óptico modelo MDT-2238A da Minipa.

$\mathrm{O}$ acoplamento entre as duas máquinas é realizado de forma direta empregando-se, para isso, um acoplamento elástico, que dispensa a necessidade de lubrificação, auxilia na acomodação de desalinhamentos e na redução dos níveis de vibração. Além disso, a fim de amenizar ruídos e vibrações, as máquinas foram instaladas sobre uma placa de aço de $1 \mathrm{~cm}$ de espessura. A referida estrutura pode ser visualizada na Figura 9.

Figura 9 - Fonte programável CSW5550.

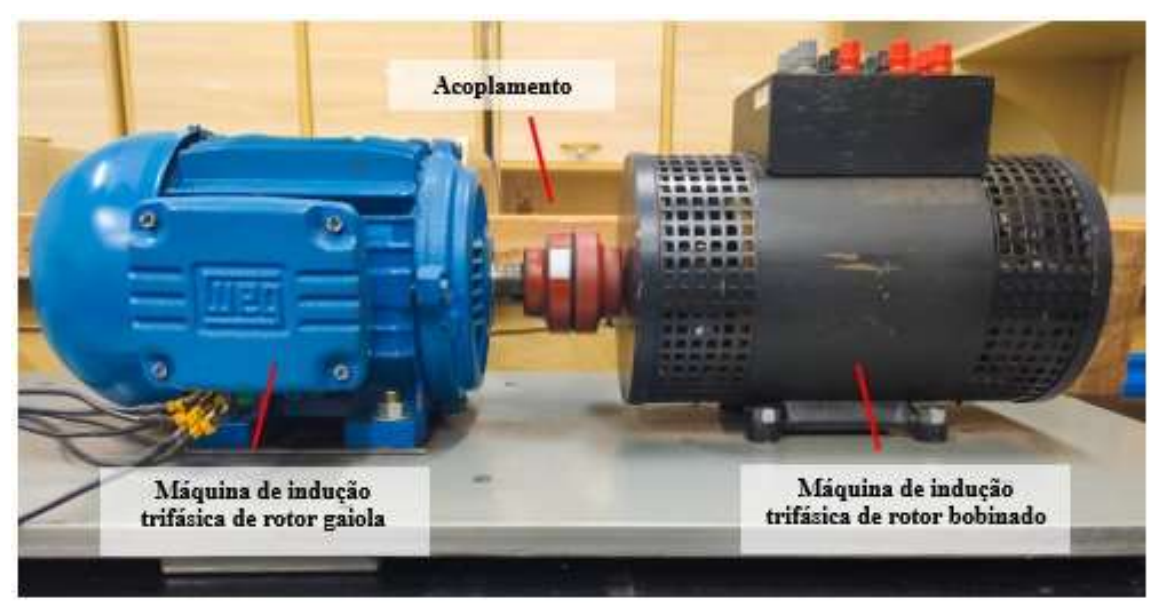

Fonte: Autores.

\subsubsection{Emulador de turbina}

Quando a MIRB é acionada como gerador, a potência ativa gerada no processo é dissipada em um banco de resistores, Figura 10, constituído por oito resistências, que podem juntas dissipar uma potência ativa total de 6,4 kW. 
Figura 10 - Banco de resistores.

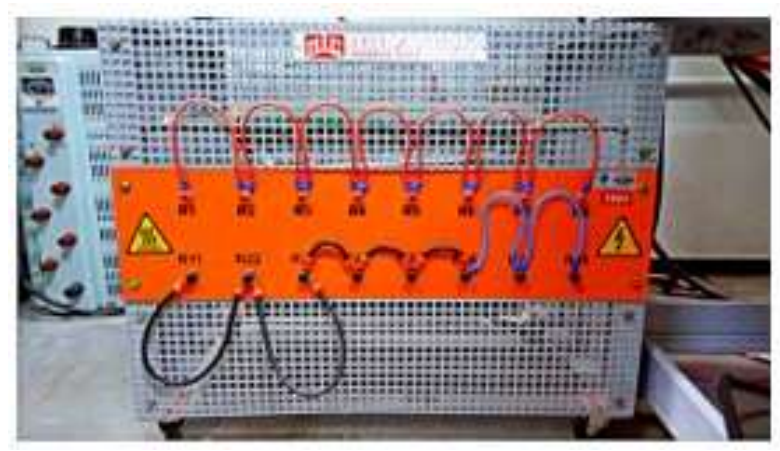

Fonte: Autores.

Na Figura 11 está apresentado o diagrama referente à ligação entre o gerador de indução de rotor bobinado (GIRB), a fonte programável e o banco de resistores. Nessa topologia de gerador ocorre a geração de potência ativa, enquanto há absorção de potência reativa por parte da máquina para manter seu campo magnético. Dessa forma, além de permitir simular uma rede elétrica com diferentes tipos de distúrbios, a fonte programável também é responsável por manter o suprimento de potência reativa e garantir o adequado funcionamento do gerador. Com relação ao banco de resistores, seu emprego tem como objetivo dissipar a potência ativa gerada e evitar que esta retorne para a fonte.

Figura 11 - Diagrama de ligação do gerador de indução de rotor bobinado conectado ao banco de resistência e à fonte programável.

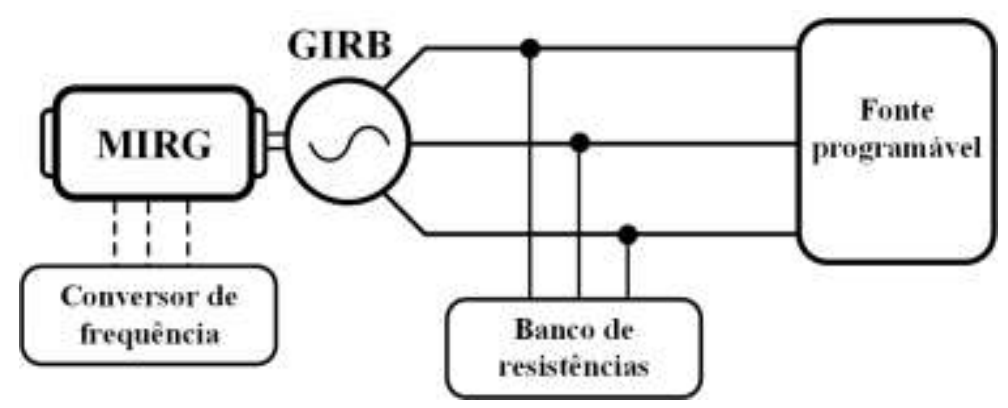

Fonte: Autores.

\subsubsection{Sistema de aquisição de dados}

Durante o acionamento da máquina de indução trifásica de rotor bobinado é necessário realizar a aquisição dos sinais instantâneos de tensão e corrente. Dessa forma, um sistema de condicionamento e aquisição de dados é essencial para mensurar, de maneira adequada, essas grandezas elétricas. A Figura 12 apresenta o diagrama em blocos do sistema implementado. 
Figura 12 - Composição do sistema de condicionamento e aquisição dos sinais de tensão e corrente.
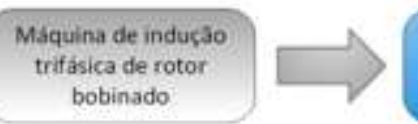

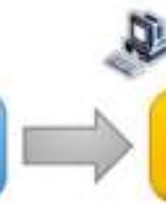

Modulo SCB - 68

condicionamento de
tensito e cotrente

Modulo SCB -68
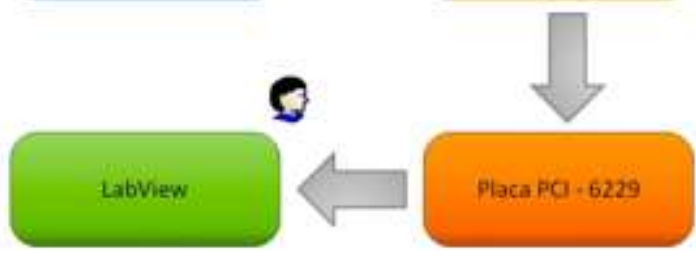

Fonte: Autores.

O condicionamento do sinal de tensão e de corrente é realizado por meio dos sensores LV20P e LA55P, respectivamente, da fabricante LEM, que trabalham segundo o princípio do efeito "hall”. Posteriormente, é empregado o módulo SCB-68 para realizar a interface entre os módulos de condicionamento e a placa de aquisição PCI-6229. Por fim, a placa PCI-6229 realiza a conversão analógica/digital dos dados mensurados pelos módulos, tornando-se possível a manipulação dos sinais no software LabView, como está mostrado na Figura 13.

Figura 13 - Tela de supervisão desenvolvida no LabView.

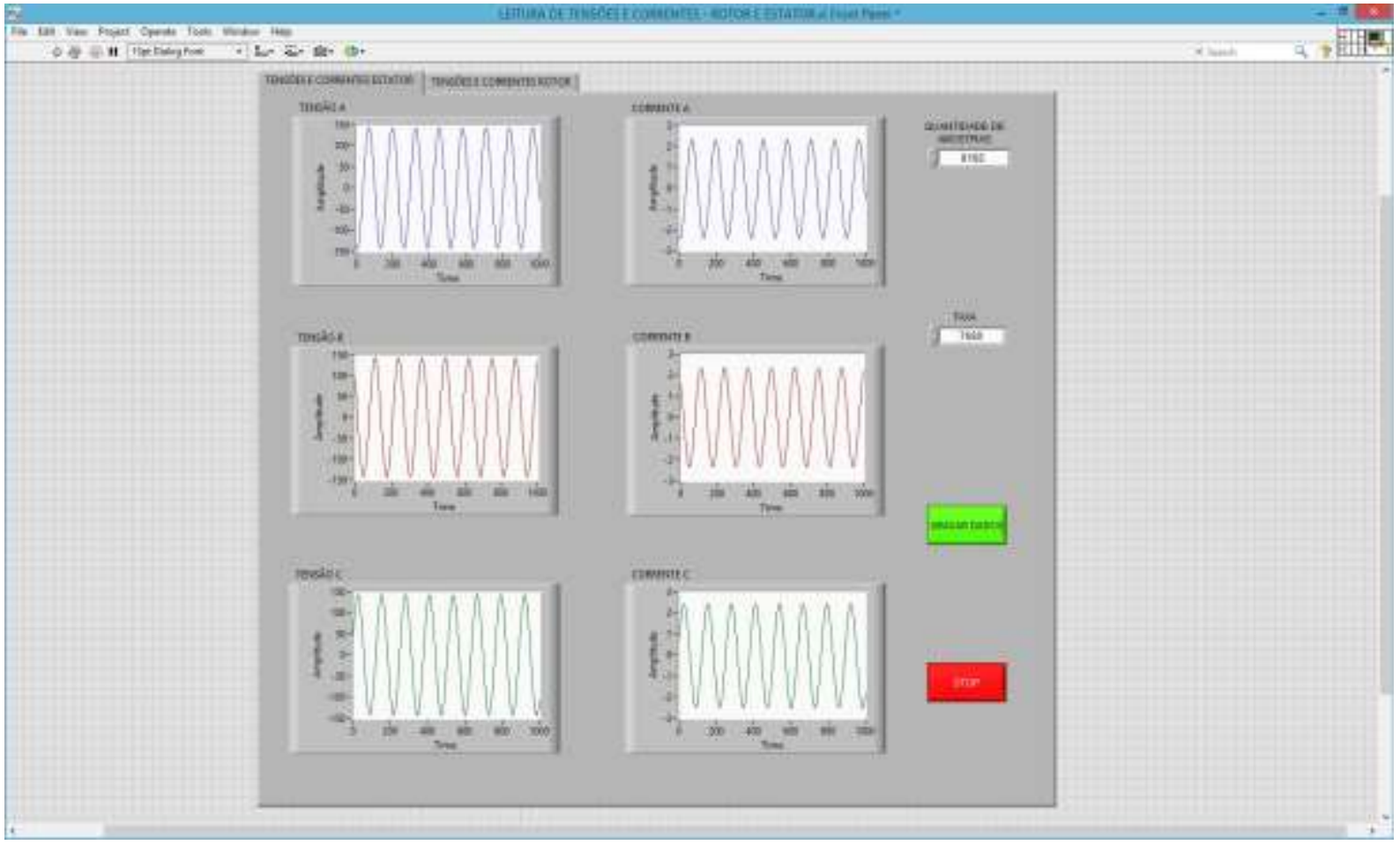

Fonte: Autores.

\subsection{Desequilíbrio de tensão}

Segundo o PRODIST, em seu módulo 8, o desequilíbrio de tensão é o fenômeno caracterizado por qualquer diferença existente nas amplitudes entre as três tensões de fase de um determinado sistema trifásico, e/ou na defasagem elétrica de $120^{\circ}$ entre as tensões de fase do mesmo sistema. 
De forma analítica, um sistema trifásico, considerando uma sequência de fase $\mathrm{ABC}$, pode ter as tensões expressas da seguinte maneira:

$$
\begin{gathered}
V_{\alpha}=\left|V_{\alpha}\right| \angle 0^{\circ} \mathrm{V} \\
V_{b}=\left|V_{b}\right| \angle-120^{\circ} \mathrm{V} \\
V_{c}=\left|V_{c}\right| \angle 120^{\circ} \mathrm{V}
\end{gathered}
$$

Assim, se o sistema é equilibrado, $\left|V_{a}\right|,\left|V_{b}\right|$ e $\left|V_{c}\right|$ possuem os mesmos valores nas expressões anteriores. Caso contrário, ou seja, se há desequilíbrio de tensão, os módulos e/ou os ângulos apresentarão valores distintos daqueles indicados em (20), (21) e (22) (Neves, 2014).

Diante disso, é definido no PRODIST o fator de desequilíbrio, grandeza responsável por indicar a porcentagem de desequilíbrio de tensão existente entre as fases de um sistema trifásico. A expressão matemática que permite seu cálculo está apresentada a seguir:

$$
F D \%=100 \cdot \sqrt{\frac{1-\sqrt{3-6 \beta}}{1+\sqrt{3-6 \beta}}}
$$

Sendo:

$F D \%$ - Fator de desequilíbrio em porcentagem.

O coeficiente $\beta$ em (23) pode ser determinado da seguinte forma:

$$
\beta=\frac{V_{a b}^{4}+V_{b c}^{4}+V_{c a}^{4}}{\left(V_{a b}^{2}+V_{b e}^{2}+V_{c a}^{2}\right)^{2}}
$$

Onde:

$V_{a b}, V_{b v}, V_{c a}-$ Módulo das tensões de linha, em valor eficaz.

A ANEEL estabelece, para tensões menores que $1 \mathrm{kV}$, o valor máximo de $3 \%$ para o fator de desequilíbrio, enquanto que, para tensões maiores que $1 \mathrm{kV}$ e menores que $230 \mathrm{kV}$, o limite passa a ser de $2 \%$.

\subsubsection{Componentes simétricas}

O método de componentes simétricas, desenvolvido por Charles Legeyt Fortescue em 1918 (Fortescue, 1918), motivo pelo qual é conhecido, também, como Teorema de Fortescue, contribui, em muito, na análise de sistemas elétricos polifásicos desequilibrados. A ideia central do método é realizar a decomposição seja da tensão ou da corrente em três diferentes componentes que, quando somadas, resultam no elemento original. Na Figura 14 está mostrado, de forma hipotética, as componentes simétricas para a tensão. 
Figura 14 - Decomposição em componentes simétricas.

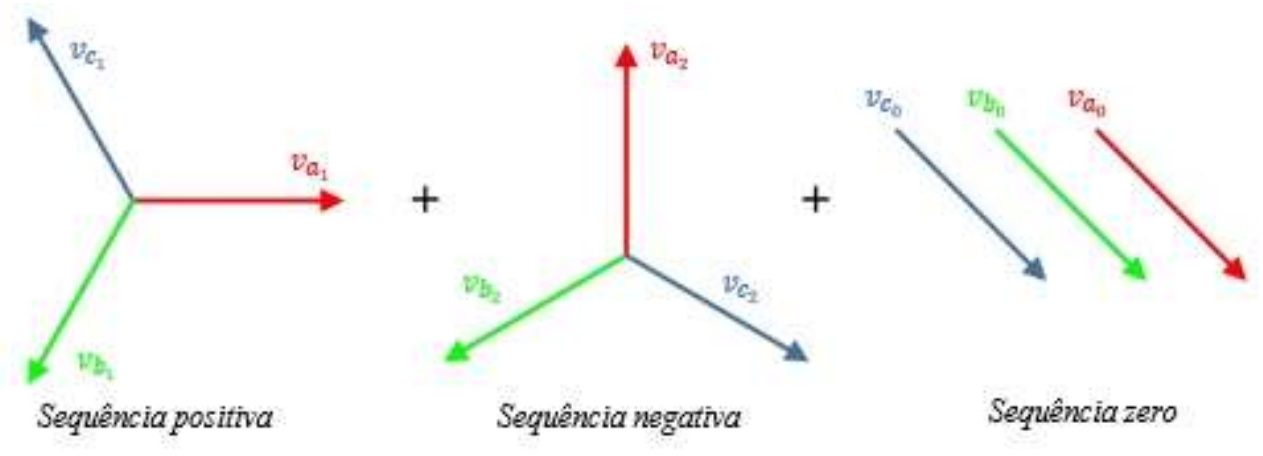

Fonte: Autores.

As componentes simétricas resultantes da decomposição do elemento original são classificadas da seguinte maneira:

- $v_{a_{1}}, v_{b_{1}}, v_{c_{1}}-$ Componente de sequência positiva que representa a componente do elemento em condições nominais equilibradas.

- $v_{C_{2}}, v_{b_{2}}, v_{C_{2}}-$ Componente de sequência negativa que representa a componente do elemento com sentido de giro inverso ao da componente de sequência positiva.

- $v_{a_{0}}, v_{b_{0}}, v_{C_{0}}-$ Componente de sequência zero que representa a componente não girante do elemento.

O sistema de equações dado por (25) relaciona as tensões de um sistema trifásico "abc" com as componentes simétricas da fase “a” (Fortescue, 2018). O mesmo procedimento é válido para as correntes.

$$
\left[\begin{array}{l}
V_{a} \\
V_{b} \\
V_{c}
\end{array}\right]=\left[\begin{array}{ccc}
1 & 1 & 1 \\
1 & a^{2} & a \\
1 & a & a^{2}
\end{array}\right] \cdot\left[\begin{array}{l}
V_{a_{1}} \\
V_{a_{1}} \\
V_{a_{2}}
\end{array}\right]
$$

Em que:

$$
a=1 \angle 120^{\circ}
$$

Efetuando a transformada inversa de (25), tem-se o conjunto de equações que permitem determinar as componentes simétricas relativas à fase "a":

$$
\left[\begin{array}{l}
V_{\mathbb{a}_{1}} \\
V_{a_{1}} \\
V_{a_{2}}
\end{array}\right]=\frac{1}{3} \cdot\left[\begin{array}{ccc}
1 & 1 & 1 \\
1 & a & a^{2} \\
1 & a^{2} & a
\end{array}\right] \cdot\left[\begin{array}{l}
V_{a} \\
V_{b} \\
V_{c}
\end{array}\right]
$$

Com as componentes simétricas da fase "a" pode-se obter as da fase "b" e "c" a partir das expressões a seguir: 


$$
\begin{array}{cc}
V_{b_{0}}=V_{a_{0}} & V_{c_{0}}=V_{a_{0}} \\
V_{b_{1}}=a^{2} \cdot V_{a_{1}} & V_{c_{1}}=a \cdot V_{a_{1}} \\
V_{b_{2}}=a \cdot V_{a_{2}} & V_{c_{2}}=a^{2} \cdot V_{a_{2}}
\end{array}
$$

Essa decomposição empregando componentes simétricas permite quantificar, em especial, a componente de sequência negativa da tensão que, no que diz respeito às máquinas de indução trifásicas, acarreta efeitos adversos que serão abordados mais profundamente no estudo de caso envolvendo desequilíbrio de tensão.

\subsubsection{Principais causas do desequilíbrio de tensão}

De acordo com Neves (2014), o desequilíbrio de tensão pode surgir, na maioria dos casos, em decorrência dos seguintes fatores:

- Distribuição irregular das cargas monofásicas entre as fases.

- Impedâncias distintas entre os enrolamentos do transformador.

- Transposição incompleta de linhas de transmissão.

- $\quad$ Falhas em banco de capacitores, como o rompimento do fusível de uma das fases.

- Motores elétricos trifásicos com impedâncias desequilibradas.

- Desigualdade nas impedâncias das linhas de transmissão.

- $\quad$ Níveis divergentes de distorção harmônica nas fases do sistema elétrico.

- $\quad$ Fenômenos como interrupções, sub-tensões, sobretensões, afundamentos, etc.

\section{Resultados e Discussões}

Para cada situação analisada são mostradas as formas de onda das tensões e correntes de estator, assim como seu espectro harmônico seja dos ensaios experimentais, quanto das simulações computacionais. Ademais, é apresentado o gráfico do conjugado eletromagnético desenvolvido pelo gerador e seu espectro harmônico.

Ainda vale ressaltar que, na simulação computacional, Figura 15, mais especificamente no equacionamento mecânico, é considerado como parâmetro de entrada a velocidade mecânica do eixo do rotor. Além disso, é importante reiterar que os enrolamentos de estator são conectados em delta-paralelo, e os de rotor são curto-circuitados externamente.

Figura 15 - Estrutura principal da simulação computacional.

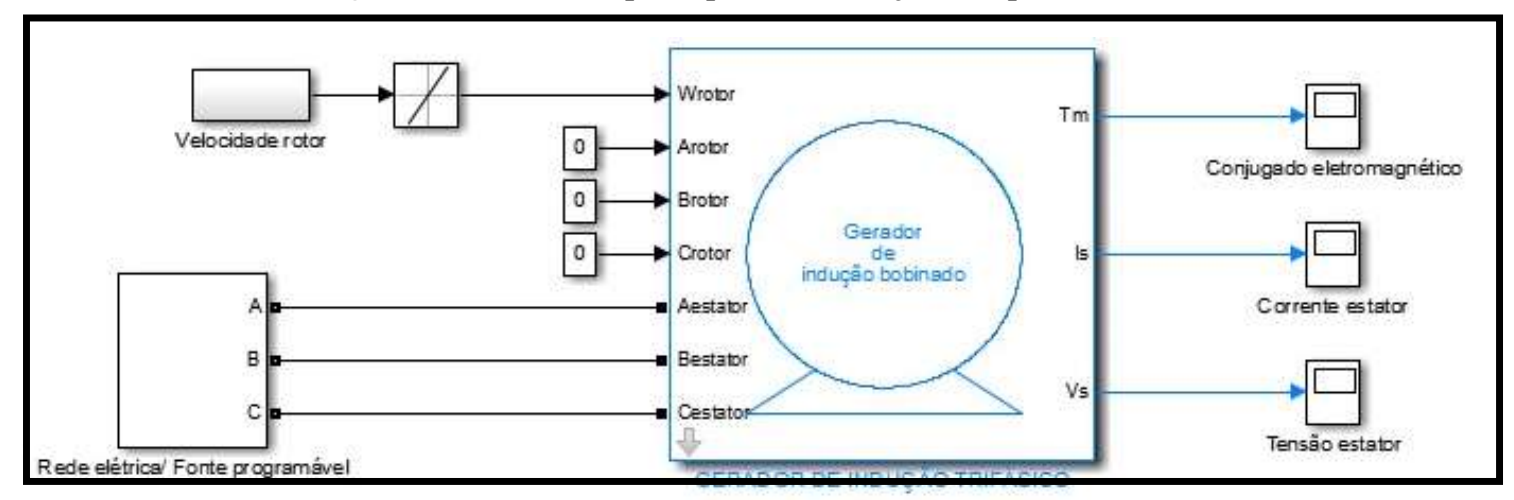

Fonte: Autores. 
Para o cálculo dos valores eficazes de tensão $\left(V_{\text {RMS }}\right)$ e corrente $\left(I_{\text {RMS }}\right)$ são empregados os valores instantâneos de tensão (v) e corrente (i) em um dado período de tempo $(T)$ nas seguintes equações (Nakashima, 2013):

$$
\begin{aligned}
& V_{\text {RMS }}=\sqrt{\frac{1}{T} \cdot \int_{0}^{T} v^{2}(t) \cdot d t} \\
& I_{\text {RMS }}=\sqrt{\frac{1}{T} \cdot \int_{0}^{T} i^{2}(t) \cdot d t}
\end{aligned}
$$

\subsection{Características magnéticas da máquina}

Antes de apresentar os resultados obtidos nos ensaios experimentais e nas simulações computacionais, são mostradas na Figura 16 as características magnéticas abordadas em 2.1, sendo elas as grandezas $F_{1}(F M)$ e $F_{9}(F M)$. Pelo fato de terem sido mensuradas as correntes de linha com os enrolamentos do estator conectados em delta-paralelo, a componente $F_{3}(F M)$ é nula. Analisando a Figura 16-(a), é possível verificar que a saturação do material ferromagnético se inicia próximo de um $\overline{F M}$ igual a 4 A.

Figura 16 - Curvas experimentais: (a) $F_{1}(\overline{F M}) \times \overline{F M}$; (b) $F_{2}(\overline{F M}) \times \overline{F M}$.

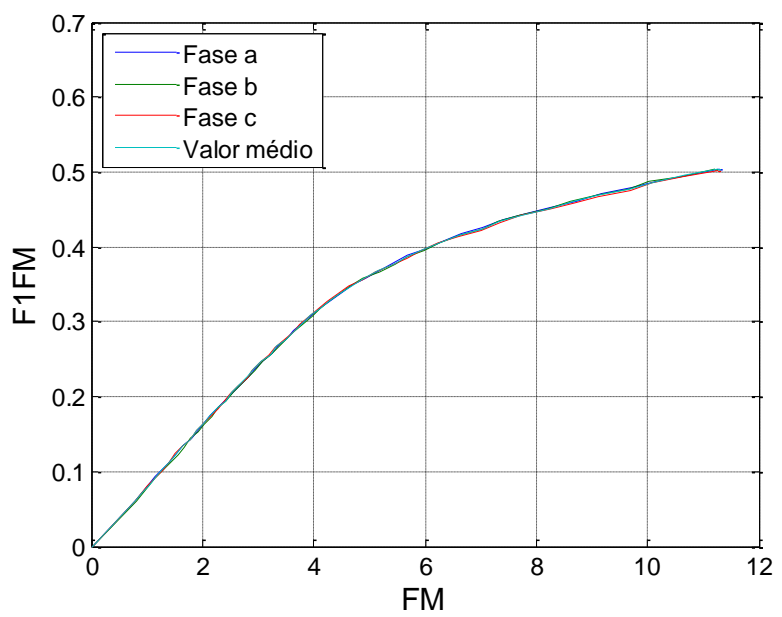

(a)

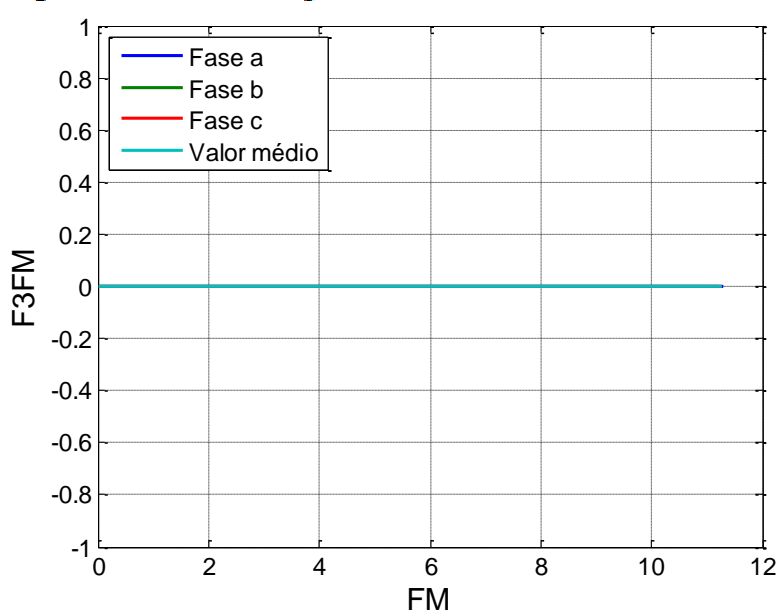

(b)

Fonte: Autores.

\subsection{Ensaio em condições normais de tensão}

Este primeiro ensaio é realizado sob condições ideais de tensão, tendo sido programado na fonte uma tensão trifásica balanceada e senoidal. Para atuar como gerador, o conversor de frequência que aciona a máquina primária é configurado para que esta opere na velocidade de $1850 \mathrm{rpm}$.

O ensaio realizado nesta etapa conduziu aos resultados que são apresentados a seguir. Nas Figuras 17-(a) e 17-(b), e nas Figuras 18-(a) e 18-(b), estão apresentados, nessa ordem, o gráfico da tensão de linha de estator e seu espectro harmônico, e o gráfico da corrente de linha de estator, do mesmo modo, com seu espectro harmônico, todos relativos ao ensaio experimental com a MIRB acionada pela máquina primária à velocidade de $1850 \mathrm{rpm}$. Como era de se esperar, tanto as tensões quanto as correntes se apresentaram equilibradas e com conteúdo harmônico na corrente praticamente desprezível. 
Figura 17 - Ensaio experimental com a rede trifásica balanceada e sem harmônicos: (a) Gráfico da tensão de linha; (b) Espectro harmônico da tensão de linha.

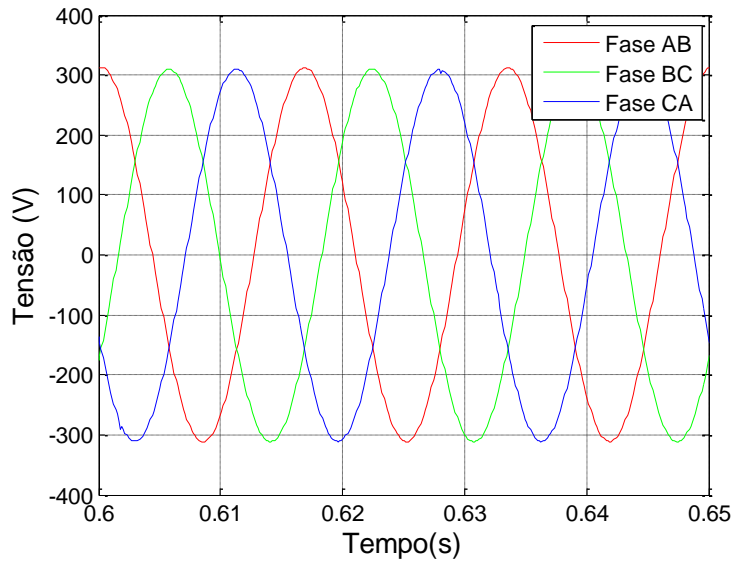

(a)

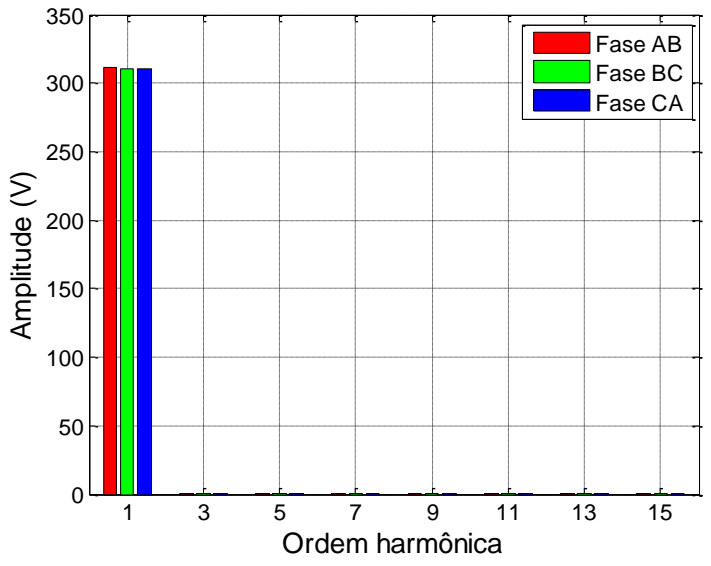

(b)

Fonte: Autores.

Figura 18 - Ensaio experimental com a rede trifásica balanceada e sem harmônicos: (a) Gráfico da corrente de linha; (b) Espectro harmônico da corrente de linha.

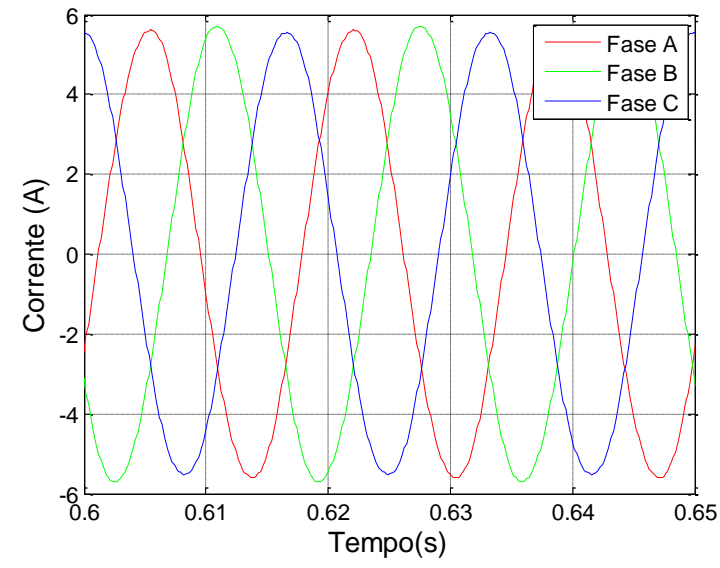

(a)

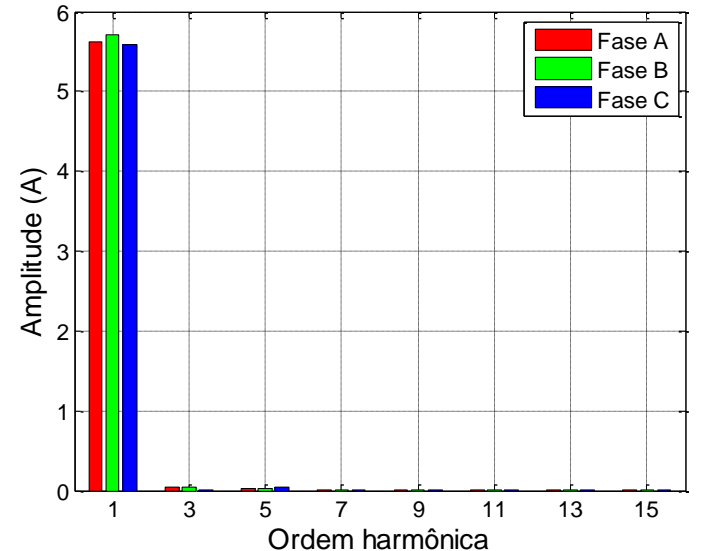

(b)

Fonte: Autores.

Os valores eficazes de tensão e corrente do gerador, sendo ambos relativos às grandezas de linha, estão apresentados na Tabela 5 .

Tabela 5 - Valores eficazes das tensões e correntes de linha relativos ao ensaio experimental.

\begin{tabular}{cccc}
\hline \multicolumn{2}{c}{ Tensão de linha } & \multicolumn{2}{c}{ Corrente de linha } \\
\hline & Valor $\left(V_{R M S}\right)$ & & Valor $\left(\boldsymbol{I}_{\text {RMS }}\right)$ \\
\hline$V_{a b}$ & 220,418 & $\boldsymbol{I}_{a}$ & 3,978 \\
\hline$V_{b c}$ & 219,518 & $\boldsymbol{I}_{b}$ & 4,037 \\
\hline$V_{c a}$ & 219,342 & $\boldsymbol{I}_{c}$ & 3,945 \\
\hline
\end{tabular}

Fonte: Autores.

A seguir são apresentados os gráficos de tensão, Figura 19-(a), e corrente, Figura 20-(a), provenientes da simulação computacional, bem como os espectros harmônicos desses sinais nas Figuras 19-(b) e 20-(b), respectivamente. 
Figura 19 - Simulação computacional com a rede trifásica balanceada e sem harmônicos: (a) Gráfico da tensão de linha; (b) Espectro harmônico da tensão de linha.

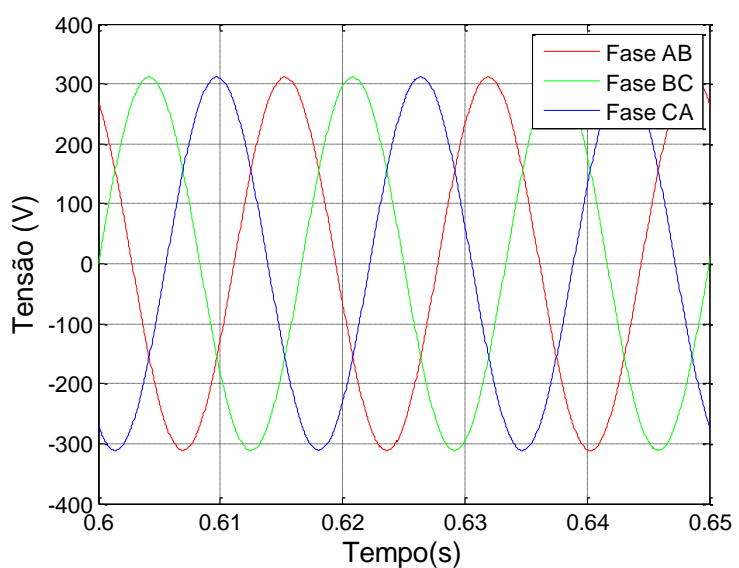

(a)

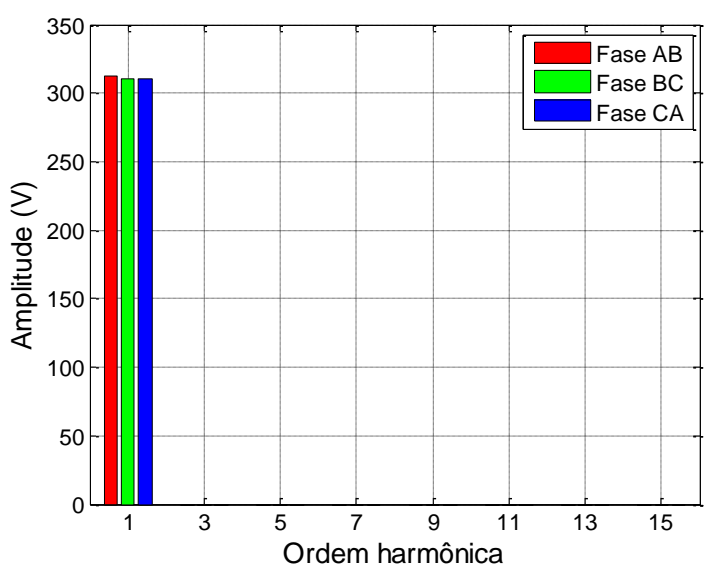

(b)

Fonte: Autores.

Figura 20 - Simulação computacional com a rede trifásica balanceada e sem harmônicos: (a) Gráfico da corrente de linha; (b) Espectro harmônico da corrente de linha.

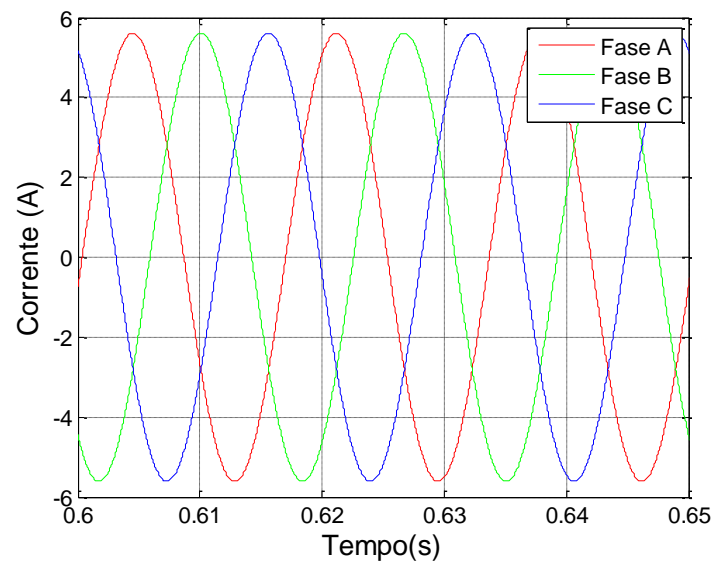

(a)

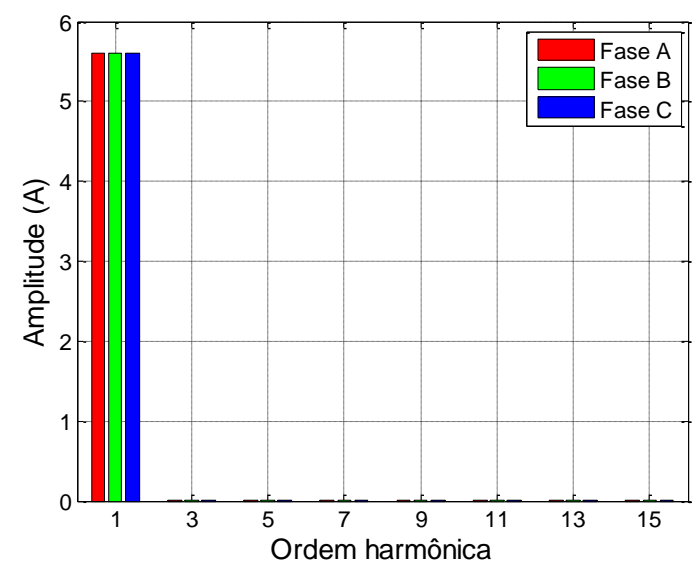

(b)

Fonte: Autores.

Os valores eficazes dos sinais de tensão e corrente apresentados anteriormente estão mostrados na Tabela 6. Comparando esses valores com os apresentados na Tabela 5, pode-se observar a semelhança entre os resultados obtidos de forma experimental e por meio da simulação computacional.

O conjugado eletromagnético, Figura 21, apresenta valor médio igual a 1,728 N.m. Um importante ponto a ser analisado é o comportamento linear do conjugado eletromagnético. Como a função harmônica magnética de terceiro harmônico não é considerada, em virtude de o ensaio para levantamento das características magnéticas ter sido realizado em delta-paralelo, conjugado eletromagnético resultante refere-se apenas à função magnética de ordem fundamental, não apresentado, assim, possíveis oscilações. 
Tabela 6 - Valores eficazes das tensões e correntes de linha relativos à simulação computacional.

\begin{tabular}{cccc}
\hline \multicolumn{2}{c}{ Tensão de linha } & \multicolumn{2}{c}{ Corrente de linha } \\
\hline & Valor $\left(V_{R M S}\right)$ & & Valor $\left(\boldsymbol{I}_{\boldsymbol{R M S}}\right)$ \\
\hline$V_{a b}$ & 220 & $\boldsymbol{I}_{\boldsymbol{a}}$ & 3,976 \\
\hline$V_{b c}$ & 220 & $\boldsymbol{I}_{b}$ & 3,976 \\
\hline$V_{c a}$ & 220 & $\boldsymbol{I}_{\boldsymbol{c}}$ & 3,976 \\
\hline
\end{tabular}

Fonte: Autores.

Figura 21 - Simulação computacional com a rede trifásica balanceada e sem harmônicos: (a) Gráfico do conjugado eletromagnético; (b) Espectro harmônico do conjugado eletromagnético.

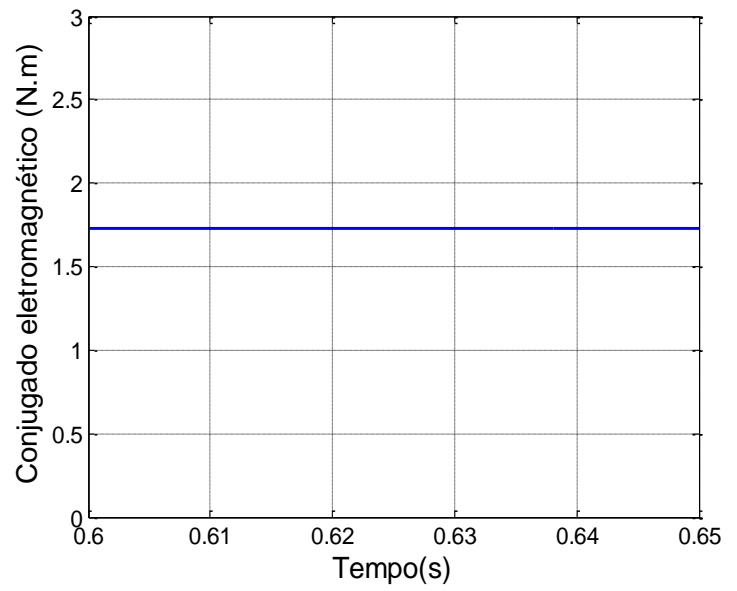

(a)

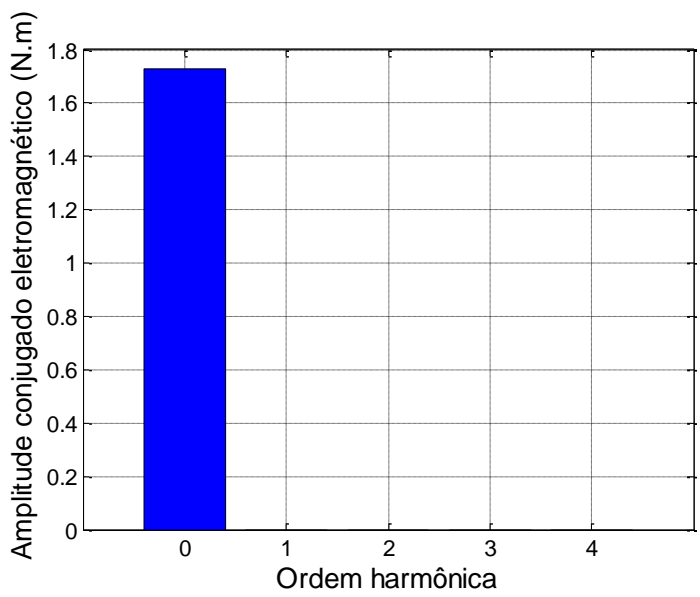

(b)

Fonte: Autores.

\subsection{Ensaio com desequilíbrio de tensão}

Primeiramente, para o estudo de caso envolvendo desequilíbrio de tensão, é necessário definir, de forma adequada, os níveis de tensão de cada uma das fases do sistema trifásico, ou seja, da rede em que o gerador está conectado. Nesse sentido, com auxílio das equações (23) e (24), foram determinados os valores de tensão que resultaram em um $F D \%$ de $2,93 \%$, respeitando o limite de 3\% estabelecido no PRODIST, como discutido em 2.3. Os valores obtidos estão apresentados na Tabela 7, os quais são utilizados como referência na programação da fonte e na simulação computacional.

Tabela 7 - Níveis de tensão para o ensaio com desequilíbrio de tensão.

\begin{tabular}{cc}
\hline & $\begin{array}{c}\text { Tensão } \\
\text { (Vrms) }\end{array}$ \\
\hline$V_{a b}$ & 229,510 \\
\hline$V_{b c}$ & 218,275 \\
\hline$V_{c a}$ & 222,663 \\
\hline
\end{tabular}

Fonte: Autores.

São apresentados a seguir os resultados para a condição de desequilíbrio de tensão à velocidade de $1850 \mathrm{rpm}$. Os gráficos de tensão e corrente de estator oriundos do ensaio experimental estão mostrados nas Figuras 22 e 23, respectivamente, e os valores eficazes na Tabela 8 . 
Figura 22 - Ensaio experimental com a rede trifásica desbalanceada e sem harmônicos: (a) Gráfico da tensão de linha; (b) Espectro harmônico da tensão de linha.

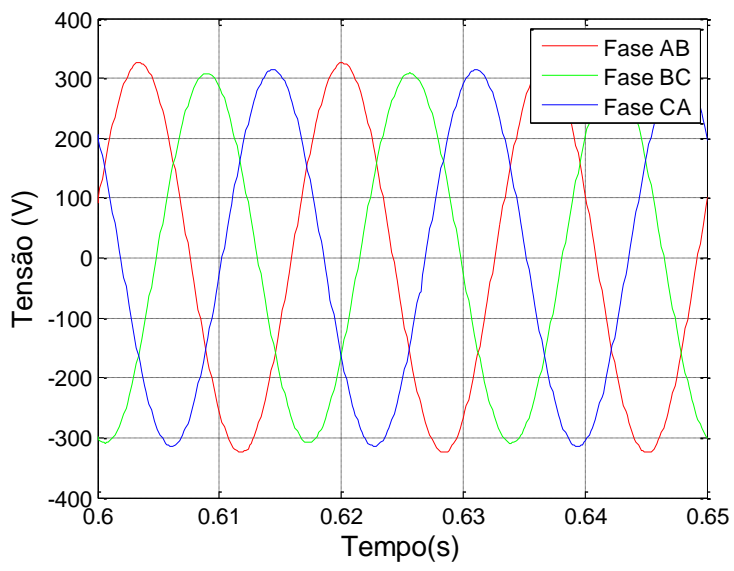

(a)

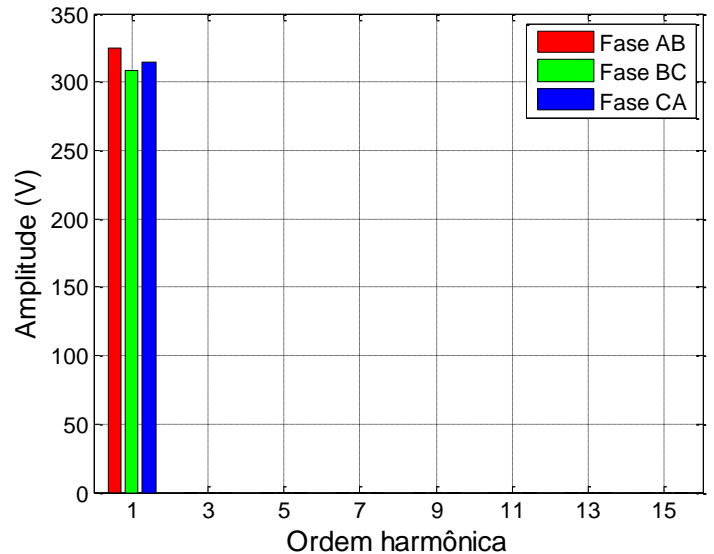

(b)

Fonte: Autores.

Figura 23 - Ensaio experimental com a rede trifásica desbalanceada e sem harmônicos: (a) Gráfico da corrente de linha; (b) Espectro harmônico da corrente de linha.

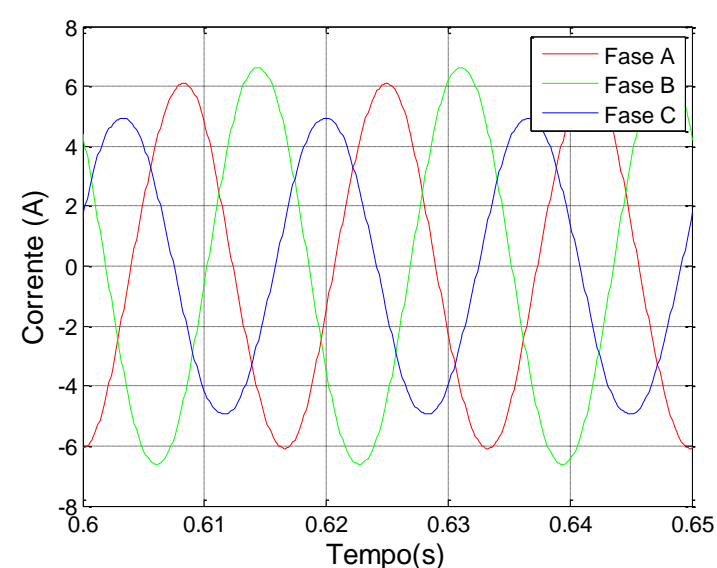

(a)

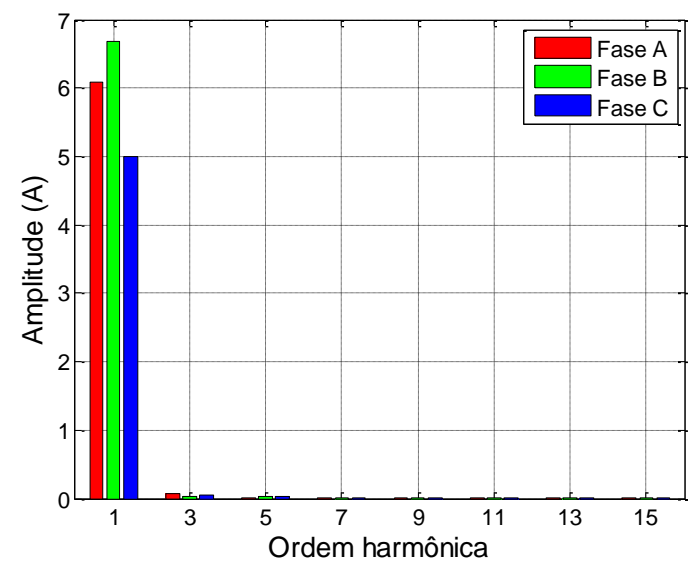

(b)

Fonte: Autores.

Tabela 8 - Valores eficazes das tensões e correntes de linha relativos à simulação computacional.

\begin{tabular}{cccc}
\hline \multicolumn{2}{c}{ Tensão de linha } & \multicolumn{2}{c}{ Corrente de linha } \\
\hline & Valor $\left(V_{R M S}\right)$ & & Valor $\left(\boldsymbol{I}_{R M S}\right)$ \\
\hline$V_{a b}$ & 229,527 & $\boldsymbol{I}_{a}$ & 4,181 \\
\hline$V_{b c}$ & 218,284 & $\boldsymbol{I}_{b}$ & 4,642 \\
\hline$V_{c a}$ & 222,668 & $\boldsymbol{I}_{c}$ & 3,564 \\
\hline
\end{tabular}

Fonte: Autores.

O mesmo comportamento verificado nas correntes de estator durante o ensaio experimental é observado nos resultados da simulação computacional, como está exposto na Figura 24, considerando a tensão da rede, Figura 25, com os mesmos valores utilizados no ajuste da fonte indicados na Tabela 7. 
Figura 24 - Simulação computacional com a rede trifásica desbalanceada e sem harmônicos: (a) Gráfico da tensão de linha; (b) Espectro harmônico da tensão de linha.

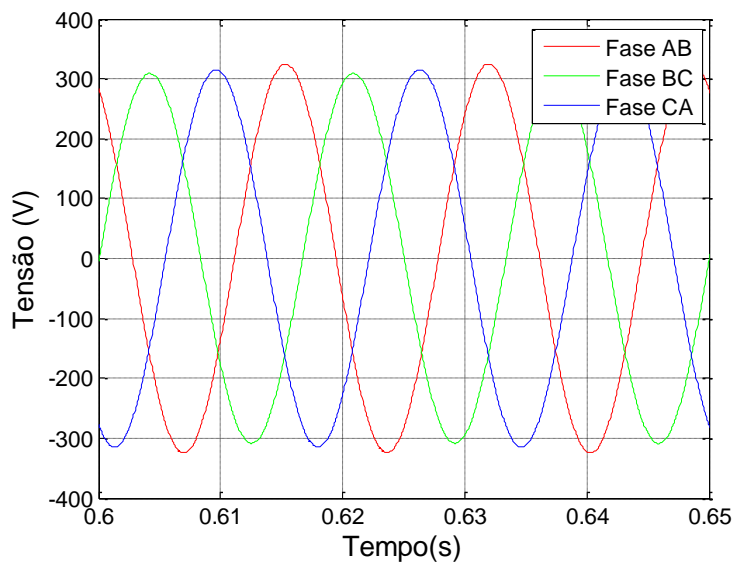

(a)

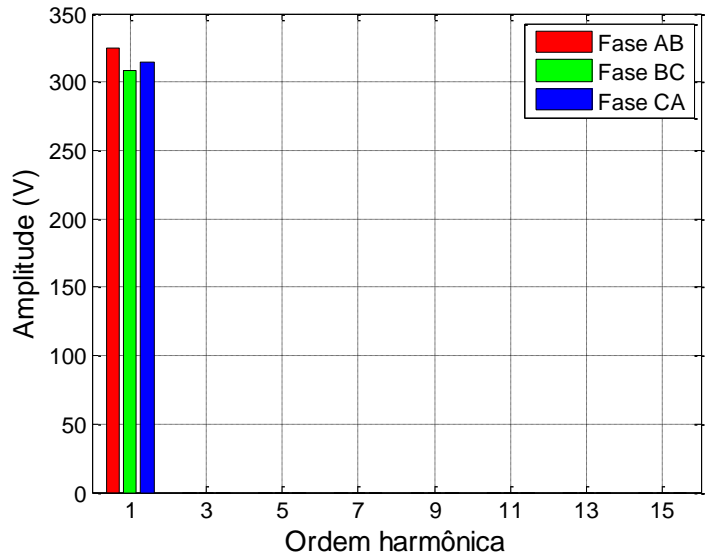

(b)

Fonte: Autores.

Figura 25 - Simulação computacional com a rede trifásica desbalanceada e sem harmônicos: (a) Gráfico da corrente de linha; (b) Espectro harmônico da corrente de linha.

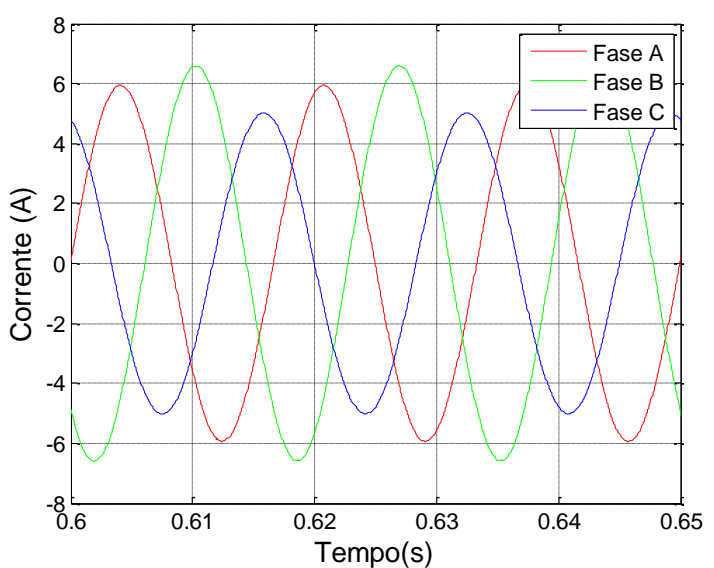

(a)

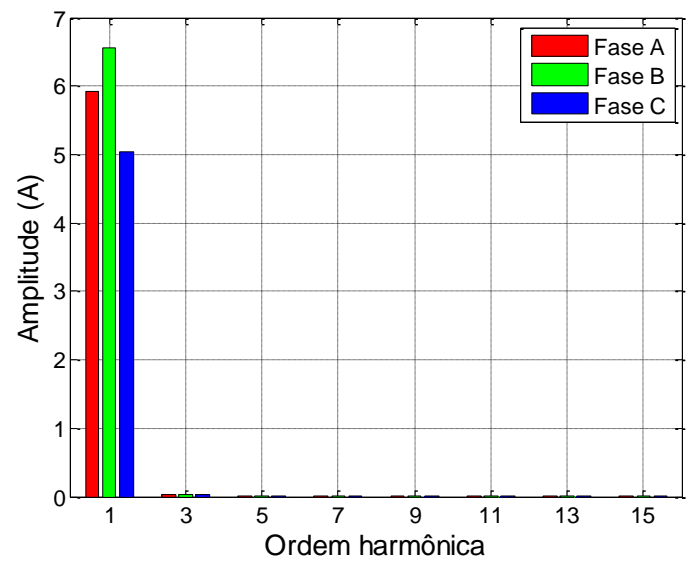

(b)

Fonte: Autores.

Comparando os resultados apresentados na Tabela 9, relativos ao estudo teórico, com aqueles apresentados na Tabela 8, pertinentes ao ensaio experimental, pode-se inferir na adequada resposta do modelo matemático da máquina de indução para situações com desequilíbrio de tensão.

Tabela 9 - Valores eficazes das tensões e correntes de linha relativos à simulação computacional.

\begin{tabular}{cccc}
\hline \multicolumn{2}{c}{ Tensão de linha } & \multicolumn{2}{c}{ Corrente de linha } \\
\hline & Valor $\left(V_{R M S}\right)$ & & Valor $\left(\boldsymbol{I}_{R M S}\right)$ \\
\hline$V_{a b}$ & 229,527 & $\boldsymbol{I}_{a}$ & 4,181 \\
\hline$V_{b c}$ & 218,284 & $\boldsymbol{I}_{b}$ & 4,642 \\
\hline$V_{c a}$ & 222,668 & $\boldsymbol{I}_{c}$ & 3,564 \\
\hline
\end{tabular}

Fonte: Autores.

No que tange ao conjugado eletromagnético, percebe-se pela Figura 26 a ocorrência de uma expressiva oscilação. De acordo com Muljadi et al. (1999) esse fenômeno é conhecido como "conjugado pulsante" e está relacionado com a presença de 
uma tensão de sequência negativa que se origina com o desequilíbrio de tensão. Dessa forma, essa componente de sequência negativa resulta em um conjugado contrário ao produzido pela de sequência positiva, o qual possui o mesmo sentido de rotação do rotor. Como consequência, surgem problemas como vibrações, aquecimento e perda de rendimento (Oliveira, 2017).

Figura 26 - Simulação computacional com a rede trifásica desbalanceada e sem harmônicos: (a) Gráfico do conjugado eletromagnético; (b) Espectro harmônico do conjugado eletromagnético.

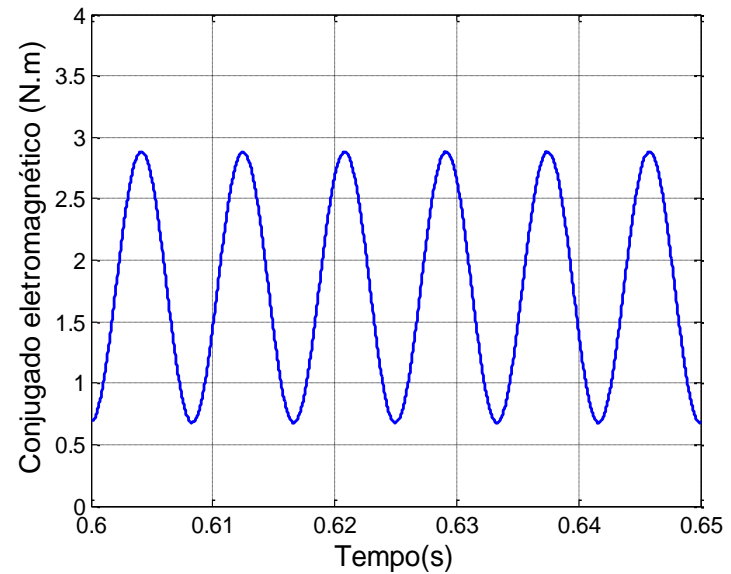

(a)

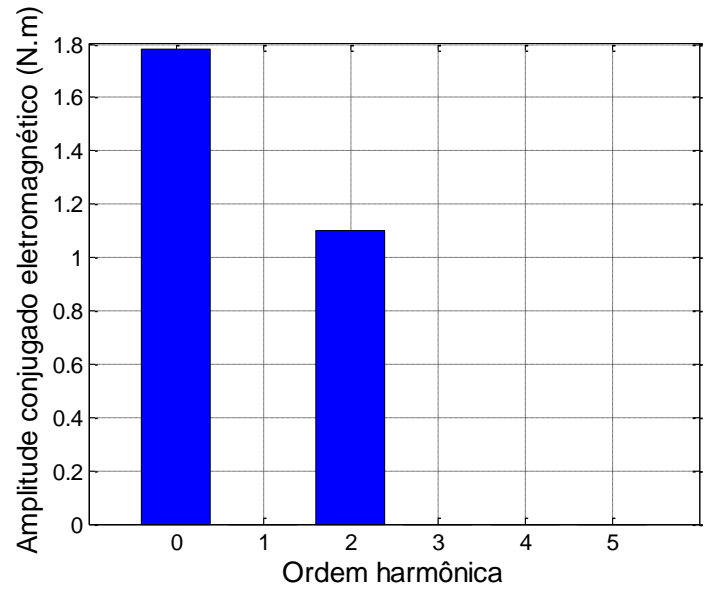

(b)

Fonte: Autores.

Pela Figura 26-(b), em que está mostrado o espectro harmônico do conjugado eletromagnético, é possível observar que esse é formado por uma componente constante, com valor igual a 1,78 N.m, dado pela harmônica de ordem zero, e por uma componente de segunda ordem, com valor de 1,101 N.m. Conforme é abordado por Donolo et al. (2016), o conjugado referente à essa componente de segunda ordem, que é classificada como sequência negativa, é característica do desequilíbrio de tensão. Para determinar a componente de sequência negativa da tensão presente no sistema trifásico dado pelas tensões apresentadas na Tabela 7, basta empregar as equações (26) e (27). Fazendo isso, obtém-se um valor, em módulo, igual a 6,556 Vrms.

\section{Conclusão}

Neste estudo foi demonstrado que o "modelo na fase" da máquina de indução trifásica para a análise do gerador de indução trifásico composto por rotor bobinado conectado à rede apresentou resultados consistentes para a sua operação em condições normais e quando submetido a desequilíbrios de tensão. Essa inferência só foi possível graças a implementação de uma plataforma de ensaios robusta, que permite realizar medições de tensão, corrente, e velocidade de forma precisa e confiável, e, assim, possibilita confrontar os resultados experimentais com aqueles obtidos de forma teórica. As descobertas apresentadas demonstram que o modelo pode ser utilizado quando se necessita de resultados precisos, uma vez que as simplificações realizadas são menores do que as efetuadas em outros modelos, e por levar em consideração as características magnéticas da máquina.

\section{Agradecimentos}

Os autores agradecem à Coordenação de Aperfeiçoamento de Pessoal de Nível Superior (CAPES), à Fundação de Amparo à Pesquisa do Estado de Minas Gerais (FAPEMIG) e ao Conselho Nacional de Desenvolvimento Científico e Tecnológico $(\mathrm{CNPq})$, pelo apoio financeiro. 


\section{Referências}

Abad, G. \& Iwanski, G. (2014). Properties and Control of a Doubly Fed Induction Machine. Power Electronics for Renewable Energy Systems, Transportation and Industrial Applications, 18, 270-318.

Bispo, D, Martins, L., Resende, J. T. \& Andrade, D. A. (2001). A New Strategy for Induction Machines Modeling Taking into Account the Magnetic Saturation. IEEE Transactions on Industry Applications, 37(6), 1710-1719.

Bim, E. (2012). Máquinas elétricas e acionamento. (2a ed.), Elsevier.

Donolo, P., Bossio, G., De Angelo, C., García, G. \& Donolo, M. (2016). Voltage unbalance and harmonic distortion effects on induction motor power, torque and vibrations. Electric power systems research, 140, 866-873.

El-Shimy, M. (2010). Optimal site matching of wind turbine generator: Case study of the Gulf of Suez region in Egypt. Renewable Energy, 35(8), No. 8, 18701878 .

Freitas, W., Vieira Jr, J. C. M., França, A. M., Silva, L. C. P. D. \& Costa, V. F. D. (2005). Análise comparativa entre geradores síncronos e geradores de indução com rotor tipo gaiola de esquilo para aplicação em geração distribuída. Controle \& Automação Sociedade Brasileira de Automatica, $16(3), 332-344$.

Fitzgerald, A. E., Kingsley, J. R. \& Umans, C. (2006). Tradução Anatólio Laschuk-. Máquinas Elétricas: Com introdução a eletrônica de potência. (6a ed.). Bookman.

Fortescue, C. L. (1918). Method of symmetrical co-ordinates applied to the solution of polyphase networks. Transactions of the American Institute of Electrical Engineers, 37(2), 1027-1140.

Global Wind Energy Council - GWEC. (2021). https://gwec.net/.

Gomes, L. C. (2008). Acionamento vetorial de motores de indução trifásicos com enfraquecimento de campo e maximização do conjugado por ampère. Tese de doutorado. Universidade Federal de Uberlândia.

Lamme, B. G. (1921). The story of the induction motor. Journal of the American Institute of Electrical Engineers, 40, $203-223$.

Li, W., Chao, P., Liang, X., Ma, J., Xu, D. \& Jin, X. (2017). A practical equivalent method for DFIG wind farms. IEEE Transactions on Sustainable Energy, $9,610-620$.

Lima, M. D. S. (2019). Uma análise crítica das distorções harmônicas de correntes associadas a aerogeradores DFIG. Dissertação de mestrado. Universidade Federal de Uberlândia.

Liu, M., Pan, W., Zhang, Y., Zhao, K., Zhang, S., \& Liu, T. (2019). A dynamic equivalent model for DFIG-based wind farms. IEEE Access, 7, 74931-74940.

Muljadi, E., Yildirim, D., Batan, T., \& Butterfield, C. P. (1999). Understanding the unbalanced-voltage problem in wind turbine generation. 1999 IEEE Industry Applications Conference, 2, 1359-1365.

Nakashima, K. (2013). Valor médio e eficaz. http://www.profelectro.info/wp-content/uploads/rms_medio_multimetro_Dc_e_AC.pdf. Acesso em 27 de out. de 2021.

Neves, A. B. F. (2014). Análise dos Efeitos do Desequilíbrio e da Distorção Harmônica de Tensão no Conjugado e no Rendimento de um Motor de Indução Trifásico. Dissertação de mestrado. Universidade de Brasília.

Oliveira, C. A. (2018). Plataforma para ensaios de motores de indução trifásicos e simulação de cargas mecânicas: acionamento, operação e monitoramento com auxílio de fonte programável. Dissertação de mestrado. Universidade Federal de Uberlândia.

Oliveira, I. A. C. (2017). Sistema de geração eólica baseado no gerador de indução de rotor bobinado alimentado por tensões desequilibradas. Dissertação de mestrado. Universidade Federal da Paraíba.

Procedimentos de Distribuição de Energia Elétrica no Sistema Elétrico Nacional. Módulo 8, Revisão 12, Resolução normativa n $794 / 2017$. http://www.aneel.gov.br/modulo-8 .

Resende, J. T. (1999). Modelagem da Máquina de Indução Trifásica incluindo a Saturação Magnética - Análise Dinâmica do Gerador de Indução AutoExcitado. Tese de doutorado. Universidade Federal de Uberlândia.

Santos, Â. M. M., Medeiros, L. T. P., Silva, L. P., Junior, I. D. S., Teixeira, V. S. D. C. \& Moreira, A. B. (2019). Wind power system connected to the grid from Squirrel Cage Induction Generator (SCIG). IEEE 15th Brazilian Power Electronics Conference and 5th IEEE Southern Power Electronics Conference, 16.

Vanço, W. E., Silva, F. B., Monteiro, J. R. B. A., Oliveira, C. M. R., Gomes, L. C. \& Carvalho, D. P. (2018). Feasibility Analysis of the Use of the Generation of Induction in the Distributed Generation. IEEE Latin America Transactions, 16, 1921-1927.

Zhou, Y., Zhao, L. \& Lee, W. J. (2018). Robustness analysis of dynamic equivalent model of DFIG wind farm for stability study. IEEE Transactions on Industry Applications, 54, 5682-569. 